\title{
Asymptotic Behavior of Solutions to Reaction-Diffusion Equations with Dynamic Boundary Conditions and Irregular Data
}

\author{
Yonghong Duan, ${ }^{1}$ Chunlei Hu ${ }^{(D)},{ }^{2}$ and Xiaojuan Chai ${ }^{2}{ }^{2}$ \\ ${ }^{1}$ Department of Mathematics, Taiyuan University, Taiyuan 030032, China \\ ${ }^{2}$ School of Mathematical Science, Anhui University, Hefei 230601, China \\ Correspondence should be addressed to Chunlei Hu; chunleihu_ahu@163.com
}

Received 13 March 2018; Revised 22 June 2018; Accepted 2 July 2018; Published 13 August 2018

Academic Editor: Nikos I. Karachalios

Copyright (C) 2018 Yonghong Duan et al. This is an open access article distributed under the Creative Commons Attribution License, which permits unrestricted use, distribution, and reproduction in any medium, provided the original work is properly cited.

This paper is concerned with the asymptotic behavior of solutions to reaction-diffusion equations with dynamic boundary conditions as well as $L^{1}$-initial data and forcing terms. We first prove the existence and uniqueness of an entropy solution by smoothing approximations. Then we consider the large-time behavior of the solution. The existence of a global attractor for the solution semigroup is obtained in $L^{1}(\bar{\Omega}, d \nu)$. This extends the corresponding results in the literatures.

\section{Introduction}

We consider the asymptotic behavior of solutions to the following parabolic equations with dynamic boundary conditions and irregular data,

$$
\begin{aligned}
u_{t}-\Delta u+f(u) & =h(x) \quad \text { in } \Omega \times \mathbb{R}^{+}, \\
u_{t}-\mu \Delta_{\Gamma} u+\frac{\partial u}{\partial \nu}+f(u) & =g(x) \quad \text { on } \Gamma \times \mathbb{R}^{+}, \\
u(x, 0) & =u_{0} \quad \text { in } \Omega,
\end{aligned}
$$

where $\Omega$ is a bounded domain in $\mathbb{R}^{N}(N \geq 3)$ with smooth boundary $\Gamma$. The first equation is the standard reactiondiffusion equation, and the second equation is the boundary equation, in which the value of $u$ is assumed to be the trace of the function $u$ defined for $x \in \Omega, \Delta_{\Gamma} u$ is the Laplace-Beltrami operator on $\Gamma[1], \mu>0$ plays the role of a surface diffusion coefficient, and $v$ is the outward normal to $\Omega$. By irregular data, we mean that $u_{0}(x), h(x) \in L^{1}(\Omega)$, and $g(x) \in L^{1}(\Gamma)$. We also assume that $f$ is $C^{0}$ and there exist positive constants $l, C_{1}, C_{2}, c_{0}>0$ and $r \geq 2$ such that

$$
\begin{array}{r}
C_{1}|s|^{r}-c_{0} \leq f(s) s \leq C_{2}|s|^{r}+c_{0} \\
\forall s \in \mathbb{R}
\end{array}
$$

$$
\begin{array}{r}
\left(f\left(x, s_{1}\right)-f\left(x, s_{2}\right)\right)\left(s_{1}-s_{2}\right) \geq-l\left|s_{1}-s_{2}\right|^{2} \\
\forall s_{1}, s_{2} \in \mathbb{R}
\end{array}
$$

Partial differential equations with dynamic boundary conditions like (1) have applications in various fields such as hydrodynamics, heat transfer theory, and thermoelasticity [2-5]. The existence and uniqueness of solutions for problem (1) have been studied extensively in various contexts; see, e.g., $[2,6-9]$. The long time behavior of solutions to (1) and related models have also aroused much interests in recent years. For autonomous equations, in [10] the existence of global attractors was derived under the assumption $\mu=$ $0, g, h=0$. Then in [11-13] the existence of global attractors and their fractal dimensions were further studied for certain semilinear reaction-diffusion equations with dynamic boundary conditions, while in $[9,14,15]$, the existence of global attractors was obtained for more general quasilinear parabolic equations with dynamic boundary conditions. For the nonautonomous case, the existence of pullback attractors for parabolic equations with dynamic boundary conditions was first obtained in [16], and then in [17-19], while the existence of uniform attractors for linear and quasilinear parabolic equations with dynamic boundary conditions was investigated in $[12,20]$. 
In the references aforementioned, the initial data and forcing terms involved are mostly assumed to be regular (belonging to $L^{2}(\Omega)$ or even $L^{\infty}$ ), and few results were known when the initial data and forcing terms are not regular, such as $L^{1}$ functions [21]. This motivates us to investigate the existence and uniqueness results as well as the large-time behavior of solutions to problem (1) with $L^{1}$ data. Due to irregular initial data and forcing terms, the usual framework for the existence and uniqueness of solutions does not work here. Also the large-time behavior for parabolic equations with $L^{1}$ data is much more involved. The less regularity of the data influences the regularity of the solutions greatly, which in turn causes some crucial difficulties in investigating the asymptotic behaviors of solutions.

In this paper, to derive the existence and uniqueness result we shall work in the framework of entropy solutions, which was first introduced in [22] for elliptic equations involving measure data and was then adapted to parabolic equations with $L^{1}$ data in [23]. We will borrow some ideas in [23, 24] and use smooth approximations to derive the existence of the entropy solution, whereas, to cope with the dynamic boundary conditions, some delicate analysis must be addressed. For the large-time behavior of the entropy solution, we prove the existence of global attractor in $L^{1}(\bar{\Omega}, d \nu)$. It is well known that to obtain global attractors, the most essential step is to derive the compactness of the semigroup, which more or less relies on certain uniform estimates in higher order spaces. Here to overcome the difficulties brought by the irregular initial data and forcing terms, we will perform some delicate Marcinkiewicz estimates on the solution and use the AubinSimon type compactness results to derive the compactness of the solution semigroup.

We mention that the existence and uniqueness results for elliptic or parabolic equations with Dirichlet boundary condition and $L^{1}$ or measure data have been studied extensively in the past years; see [24-26] and large amount of references therein. The large-time behaviors for parabolic equations with Dirichlet boundary conditions involving irregular data have also been studied by many authors; see, e.g., [27-29]. The results obtained here might be viewed as an extension of the results therein to problems with more general boundary conditions.

The rest of this paper is organized as follows. In Section 2, we provide some preliminaries and the main results of this paper. Then in Sections 3 and 4, we provide the proof for the main results. For convenience, in the following we use $c_{i}, C$, $C_{i}$ to denote generic constants in various occasions, and we will denote $\Omega \times(0, T), \Gamma \times(0, T)$ as $Q_{T}$ and $\Gamma_{T}$, respectively. For a function $u$ on $Q_{T}$ and $\Gamma_{T}$, we set $\{\Omega(u \geq k)\}=\{(x, t) \in$ $\left.Q_{T}: u(x, t) \geq k\right\},\{\Gamma(u \geq k)\}=\left\{(x, t) \in \Gamma_{T}: u(x, t) \geq k\right\}$.

\section{Preliminaries and the Main Result}

To deal with the dynamic boundary conditions, we introduce the Lebesgue spaces as follows (see [14] for more details). Let $\Omega \subset \mathbb{R}^{N}$ be a bounded domain with smooth boundary $\Gamma$. For $1 \leq p<1$, define the Lebesgue space $L^{p}(\bar{\Omega}, d \nu)$ as

$$
L^{p}(\bar{\Omega}, d \nu)=\left\{U=(u, v): u \in L^{p}(\Omega), v \in L^{p}(\Gamma)\right\},
$$

with norm

$$
\|U\|_{L^{p}(\bar{\Omega}, d v)}^{p}=\int_{\Omega}|u|^{p} d x+\int_{\Gamma}|v|^{p} d S,
$$

where $d \nu=\left.\left.d x\right|_{\Omega} \oplus d S\right|_{\Gamma}$ on $\bar{\Omega}$ is defined by $\nu(A)=|A \cap \Omega|+$ $S(A \cap \Gamma)$ for any measurable set $A \subset \bar{\Omega}$. Define the Sobolev space $H^{1}(\bar{\Omega}, d \nu)$ as

$$
H^{1}(\bar{\Omega}, d v)=\left\{U:=(u, v): u \in H^{1}(\Omega), v \in H^{1}(\Gamma)\right\}
$$

with norm

$$
\|U\|_{H^{1}(\bar{\Omega}, d v)}^{2}=\int_{\Omega}|\nabla u|^{2} d x+\int_{\Gamma}\left(\left|\nabla_{\Gamma} u\right|^{2}+c|u|^{2}\right) d S
$$

for $c>0$.

It is easy to see that we can identify $H^{1}(\bar{\Omega}, d \nu)$ with $H^{1}(\Omega) \oplus$ $H^{1}(\Gamma)$ under this norm. Hereafter, we denote by $H^{-1}(\bar{\Omega}, d \nu)$ the dual space of $H^{1}(\bar{\Omega}, d \nu)$.

Let $\varphi_{k}$ be the truncation at height $k \geq 0$,

$$
\varphi_{k}(s)= \begin{cases}k & s>k \\ s & |s| \leq k \\ -k & s<-k\end{cases}
$$

and denote $\Psi_{k}: \mathbb{R} \longmapsto \mathbb{R}^{+}$as its primitive function, i.e.,

$$
\Psi_{k}(s)=\int_{0}^{s} \varphi_{k}(\tau) d \tau= \begin{cases}\frac{s^{2}}{2} & |s| \leq k, \\ k|s|-\frac{k^{2}}{2} & |s| \geq k .\end{cases}
$$

It is obvious that $\Psi_{k}(s) \geq 0$ and $\Psi_{k}(s) \leq k|s|$.

We work within the framework of entropy solutions defined as follows.

Definition 1. A function $u$ is called an entropy solution of problem (1), if for any $T>0$ and $k>0, u \in$ $C\left(0, T ; L^{1}(\bar{\Omega}, d \nu)\right) \cap L^{r-1}\left(0, T ; L^{r-1}(\bar{\Omega} ; d \nu)\right)$; moreover,

$$
\begin{aligned}
& \int_{\Omega} \Psi_{k}(u-\phi)(T) d x-\int_{\Omega} \Psi_{k}(u-\phi)(0) d x \\
& \quad+\int_{\Gamma} \Psi_{k}(u-\phi)(T) d S-\int_{\Gamma} \Psi_{k}(u-\phi)(0) d S \\
& \quad+\int_{Q_{T}} \phi_{t} \varphi_{k}(u-\phi) d x d t+\int_{\Gamma_{T}} \phi_{t} \varphi_{k}(u-\phi) d S d t \\
& \quad+\int_{Q_{T}} f(u) \varphi_{k}(u-\phi) d x d t \\
& \quad+\int_{\Gamma_{T}} f(u) \varphi_{k}(u-\phi) d S d t \\
& \quad+\int_{Q_{T}} \nabla u \nabla \varphi_{k}(u-\phi) d x d t \\
& \quad+\mu \int_{\Gamma_{T}} \nabla_{\Gamma} u \nabla_{\Gamma} \varphi_{k}(u-\phi) d S d t \\
& \leq \int_{Q_{T}} h \varphi_{k}(u-\phi) d x d t+\int_{\Gamma_{T}} g \varphi_{k}(u-\phi) d S d t,
\end{aligned}
$$

for all $k>0$ and $u \in C\left(0, T ; L^{2}(\bar{\Omega}, d \nu)\right) \cap L^{\infty}\left(0, T ; L^{\infty}(\bar{\Omega} ; d \nu)\right)$ such that $\phi_{t} \in L^{2}\left(0, T ; H^{-1}(\bar{\Omega}, d \nu)\right)$. 
Our first result concerns on the existence and uniqueness of entropy solutions.

Theorem 2. Under assumptions (2)-(3) and suppose that $u_{0}, h \in L^{1}(\Omega)$ and $g \in L^{1}(\Gamma)$, there exists a unique entropy solution for problem (1).

To give the second result on the long time behavior of solutions, we recall the definition of global attractors.

Definition 3 (see [30]). Let $\{S(t)\}_{t \geq 0}$ be a semigroup on a Banach space $X$. A subset $\mathscr{A} \subset X$ is called a global attractor for the semigroup if $\mathscr{A}$ is compact in $X$ and enjoy the following properties:

(i) $\mathscr{A}$ is invariant, i.e., $S(t) \mathscr{A}=\mathscr{A}$ for any $t \geq 0$;

(ii) $\mathscr{A}$ attracts every bounded subset of $X$, i.e., for any bounded subset $B$ of $X$ and any neighborhood $\mathcal{N}(\mathscr{A})$ of the set $\mathscr{A}$, there exists a $T_{0}=T_{0}(B, \mathscr{N}(\mathscr{A}))$ such that

$$
S(t) B \subset \mathscr{N}(\mathscr{A}) \quad \text { for } t \geq T_{0} .
$$

Theorem 4. Assume that $u_{0}, h \in L^{1}(\Omega), g \in L^{1}(\Gamma)$ and $f$ satisfies assumptions (2)-(3); then the semigroup generated by problem (1) admits a global attractor $\mathscr{A}$ in $L^{1}(\bar{\Omega}, d v)$; i.e., $\mathscr{A}$ is compact, invariant in $L^{1}(\bar{\Omega}, d v)$ and attracts every bounded subset of $L^{1}(\bar{\Omega}, d \nu)$ in the norm topology of $L^{1}(\bar{\Omega}, d \nu)$.

To prove the theorems above, let us first provide some preliminaries.

For $s \in \mathbb{R}$, define the Marcinkiewicz space $M^{s}(\bar{\Omega}, d \nu)$ as the set of measurable functions $v$ such that

$$
\int_{\{\Omega(|v|>k)\}} k^{s} d x+\int_{\{\Gamma(|v|>k)\}} k^{s} d S<C,
$$

for some positive constant $C$ and all $k>0$. We have the following.

Lemma 5. Let $r, s$ be positive constants such that $r-s>0$ and let $u(x, t)$ be a function defined on $\left(Q_{T}, d \nu\right)$. If $u \in M^{r}\left(Q_{T}, d \nu\right)$, then $|u|^{s} \in L^{1}\left(Q_{T}, d v\right)$. In particular, $M^{r}\left(Q_{T}, d v\right) \quad \subset$ $L^{s}\left(Q_{T}, d \nu\right)$ for all $s, r \geq 1$ such that $r-s>0$.

The following is the well-known Aubin-Simon compactness result.

Lemma 6 (see [31]). Assume $X \subset Z \subset Y$ with compact imbedding $X \hookrightarrow Z$ ( $X, Y$ and $Z$ are Banach spaces). Let $F$ be bounded in $L^{p}(0, T ; X)$, where $1 \leq p<\infty$, and $\partial F / \partial t=$ $\{\partial f / \partial t: f \in F\}$ be bounded in $L^{1}(0, T ; Y)$. Then $F$ is relatively compact in $L^{p}(0, T ; Z)$.

\section{Existence and Uniqueness of Entropy Solutions}

In this section, we provide the proof for Theorem 2. We begin with the existence and uniqueness results for the problem with regular data.
Definition 7. Assume that $u_{0}, h(x) \in L^{2}(\Omega), g(x) \in L^{2}(\Gamma)$ and $f \in C^{0}$ satisfies (2)-(3). A function $u$ is called a weak solution of problem (1), if for any $T>0$, and $u \in C\left(0, T ; L^{2}(\bar{\Omega}, d \nu)\right) \cap$ $L^{r}\left(0, T ; L^{r}(\bar{\Omega}, d \nu)\right) \cap L^{2}\left(0, T ; H^{1}(\bar{\Omega}, d \nu)\right)$, and moreover

$$
\begin{aligned}
\int_{\Omega} & u_{t} \varphi d x+\int_{\Omega} \nabla u \nabla \varphi d x+\int_{\Gamma} u_{t} \varphi d S+\mu \int_{\Gamma} \nabla_{\Gamma} u \nabla_{\Gamma} \varphi d S \\
& +\int_{\Gamma} f(u) \varphi d S+\int_{\Omega} f(u) \varphi d x \\
= & \int_{\Omega} h(x) \varphi d x+\int_{\Gamma} g(x) \varphi d s,
\end{aligned}
$$

for all $\varphi \in C_{c}^{\infty}(\bar{\Omega})$.

Theorem 8. Assume that $u_{0}, h(x) \in L^{2}(\Omega), g(x) \quad \epsilon$ $L^{2}(\Gamma), f \in C^{0}$ satisfies (2)-(3). Then problem (1) admits a weak solution $u \in L^{2}\left(0, T ; H^{1}(\bar{\Omega}, d v)\right) \cap C\left(0, T ; L^{2}(\bar{\Omega}, d v)\right) \cap$ $L^{r}\left(0, T ; L^{r}(\bar{\Omega}, d \nu)\right)$.

Proof. The proof of this theorem is based on the standard Galerkin approximation method as in [10]; we thus omit the details for concision.

Proof of Theorem 2. Now provide the proof of the existence and uniqueness of entropy solutions for problem (1). For simplicity, we assume that $\mu=1$. Let $\left\{h^{n}\right\} \subset L^{\infty}(\Omega),\left\{g^{n}\right\} \subset$ $L^{\infty}(\Gamma)$, and $u_{0}^{n} \subset L^{\infty}(\Omega)$ be three sequences of functions strongly convergent, respectively, to $h$ in $L^{1}(\Omega)$, to $g$ in $L^{1}(\Gamma)$ and to $u_{0}$ in $L^{1}(\Omega)$ such that

$$
\begin{aligned}
\left\|u_{0}^{n}\right\|_{L^{1}(\Omega)} & \leq\left\|u_{0}\right\|_{L^{1}(\Omega)}, \\
\left\|h^{n}\right\|_{L^{1}(\Omega)} & \leq\|h\|_{L^{1}(\Omega)}, \\
\left\|g^{n}\right\|_{L^{1}(\Gamma)} & \leq\|g\|_{L^{1}(\Gamma)} .
\end{aligned}
$$

Let us consider the approximation problem of (1),

$$
\begin{aligned}
u_{t}^{n}-\Delta u^{n}+f\left(u^{n}\right) & =h^{n} & & \text { in } \Omega \times \mathbb{R}^{+}, \\
u_{t}^{n}-\Delta_{\Gamma} u^{n}+\frac{\partial u^{n}}{\partial \nu}+f\left(u^{n}\right) & =g^{n} & & \text { on } \Gamma \times \mathbb{R}^{+}, \\
u^{n}(x, 0) & =u_{0}^{n} & & \text { in } \Omega .
\end{aligned}
$$

By virtue of Theorem 8 , there is an unique weak solution $u^{n}$ to (15) for each $n$, with

$$
\begin{aligned}
u \in C & \left([0, T] ; L^{2}(\bar{\Omega}, d \nu)\right) \cap L^{r}\left(0, T ; L^{r}(\bar{\Omega} ; d \nu)\right) \\
& \cap L^{2}\left(0, T ; H^{1}(\bar{\Omega} ; d \nu)\right) .
\end{aligned}
$$

Next, we shall follow the ideas of [24] to prove that, up to a subsequence, $u^{n}$ converges to a measurable function $u$, which is the entropy solution of problem (1). Let us divide the proof into several steps. Hereafter, without indication all the convergence should be understood in the sense of subsequences. 
Step 1. $u^{n}$ converges to $u$ in $L^{1}\left(0, T ; L^{1}(\bar{\Omega}, d \nu)\right)$.

Taking $\varphi_{k}\left(u^{n}\right)(k \geq 1)$ as a test function in (15), we deduce that

$$
\begin{aligned}
& \frac{d}{d t}\left(\int_{\Omega} \Psi_{k}\left(u^{n}\right)(t) d x+\int_{\Gamma} \Psi_{k}\left(u^{n}\right)(t) d S\right) \\
& \quad+\int_{\Omega}\left|\nabla \varphi_{k}\left(u^{n}\right)\right|^{2} d x+\int_{\Gamma}\left|\nabla_{\Gamma} \varphi_{k}\left(u^{n}\right)\right|^{2} d S \\
& \quad+\int_{\Omega} f\left(u^{n}\right) \varphi_{k}\left(u^{n}\right) d x+\int_{\Gamma} f\left(u^{n}\right) \varphi_{k}\left(u^{n}\right) d S \\
& \quad \leq k\|h\|_{L^{1}(\Omega)}+k\|g\|_{L^{1}(\Gamma)} .
\end{aligned}
$$

Since

$$
\begin{aligned}
& f\left(u^{n}\right) \varphi_{k}\left(u^{n}\right)=f\left(u^{n}\right) \operatorname{sgn}\left(u^{n}\right)\left|\varphi_{k}\left(u^{n}\right)\right| \\
& \quad \geq\left(C_{1}\left|u^{n}\right|^{r-1}-C\right)\left|\varphi_{k}\left(u^{n}\right)\right| \\
& \frac{d}{d t}\left(\int_{\Omega} \Psi_{k}\left(u^{n}\right)(t) d x+\int_{\Gamma} \Psi_{k}\left(u^{n}\right)(t) d S\right) \\
& \quad+\int_{\Omega}\left|\nabla \varphi_{k}\left(u^{n}\right)\right|^{2} d x+\int_{\Gamma}\left|\nabla_{\Gamma} \varphi_{k}\left(u^{n}\right)\right|^{2} d S \\
& \quad+C_{1}\left(\int_{\Omega}\left|u^{n}\right|^{r-1}\left|\varphi_{k}\left(u^{n}\right)\right| d x\right. \\
& \left.\quad+\int_{\Gamma}\left|u^{n}\right|^{r-1}\left|\varphi_{k}\left(u^{n}\right)\right| d S\right) \leq k\|h\|_{L^{1}(\Omega)}+k\|g\|_{L^{1}(\Gamma)} \\
& \quad+C(|\Omega|+|\Gamma|) .
\end{aligned}
$$

Note that $r \geq 2$. From the definition of $\Psi_{k}(\cdot)$ we obtain

$$
\begin{aligned}
0 \leq & \int_{\Omega} \Psi_{1}\left(u^{n}\right) d x+\int_{\Gamma} \Psi_{1}\left(u^{n}\right) d S \\
\leq & \int_{\Omega}\left|u^{n}\right|\left|\varphi_{1}\left(u^{n}\right)\right| d x+\int_{\Gamma}\left|u^{n}\right|\left|\varphi_{1}\left(u^{n}\right)\right| d S \\
\leq & \int_{\Omega}\left|u^{n}\right|^{r-1}\left|\varphi_{1}\left(u^{n}\right)\right| d x+\int_{\Gamma}\left|u^{n}\right|^{r-1}\left|\varphi_{1}\left(u^{n}\right)\right| d S \\
& +|\Omega|+|\Gamma| .
\end{aligned}
$$

If we choose $k=1$ and taking the above inequality in consideration, we deduce from (19) that

$$
\begin{aligned}
& \frac{d}{d t}\left(\int_{\Omega} \Psi_{1}\left(u^{n}\right)(t) d x+\int_{\Gamma} \Psi_{1}\left(u^{n}\right)(t) d S\right) \\
& \quad+C_{1}\left(\int_{\Omega} \Psi_{1}\left(u^{n}\right)(t) d x+\int_{\Gamma} \Psi_{1}\left(u^{n}\right)(t) d S\right) \\
& \quad \leq\|h\|_{L^{1}(\Omega)}+\|g\|_{l^{1}(\Gamma)}+C|\Omega|+|\Gamma|=C_{5} .
\end{aligned}
$$

By the standard Gronwall's inequality, we obtain that

$$
\left\|\Psi_{1}\left(u^{n}\right)(t)\right\|_{L^{1}(\bar{\Omega}, d v)} \leq \frac{C_{5}}{c_{1}}+e^{-C_{1} t}\left\|\Psi_{1}\left(u_{0}^{n}\right)\right\|_{L^{1}(\bar{\Omega}, d v)} .
$$

Note that

$$
\begin{aligned}
& \int_{\Omega} \Psi_{1}\left(u^{n}\right)(t) d x+\int_{\Gamma} \Psi_{1}\left(u^{n}\right)(t) d S \\
& \quad=\left\|\Psi_{1}\left(u^{n}\right)(t)\right\|_{L^{1}(\bar{\Omega}, d v)} \\
& \quad \leq \int_{\Omega} \Psi_{1}\left(u^{n}\right)(t) d x+\int_{\Gamma} \Psi_{1}\left(u^{n}\right)(t) d S+|\Omega|+|\Gamma| .
\end{aligned}
$$

By the definition of $\Psi_{k}$, we have

$$
\begin{aligned}
\int_{\Omega} \Psi_{1}\left(u^{n}\right)(t) d x & \leq\left\|u^{n}(t)\right\|_{L^{1}(\Omega)} \\
& \leq \int_{\Omega} \Psi_{1}\left(u^{n}\right)(t) d x+|\Omega|, \\
\int_{\Gamma} \Psi_{1}\left(u^{n}\right)(t) d S & \leq\left\|u^{n}(t)\right\|_{L^{1}(\Gamma)} \\
& \leq \int_{\Gamma} \Psi_{1}\left(u^{n}\right)(t) d S+|\Gamma|
\end{aligned}
$$

Therefore, we get

$$
\left\|u^{n}(t)\right\|_{L^{1}(\bar{\Omega}, d v)} \leq \frac{C_{5}}{c_{1}}+e^{-C_{1} t}\left\|u_{0}^{n}\right\|_{L^{1}(\bar{\Omega}, d v)}+|\Omega|+|\Gamma|
$$

for any $t>0$.

Furthermore, integrating (19) between 0 and $T$, it is easy to obtain

$$
\begin{array}{r}
\int_{Q_{T}}\left|\nabla \varphi_{k}\left(u^{n}\right)\right|^{2} d x d t+\int_{\Gamma_{T}}\left|\nabla_{\Gamma} \varphi_{k}\left(u^{n}\right)\right|^{2} d S d t \leq C_{6} k, \\
\int_{Q_{T}}\left|u^{n}\right|^{r-1} d x d t+\int_{\Gamma_{T}}\left|u^{n}\right|^{r-1} d S d t \leq C_{7} .
\end{array}
$$

Setting $\lambda=2 / r$ and using (27), we deduce that

$$
\begin{aligned}
& \int_{\left\{\Omega\left(\left|\nabla u^{n}\right|^{\lambda}>k\right)\right\}} k^{r-1} d x d t \\
&=\int_{\left\{\left|\nabla u^{n}\right|^{\lambda}>k \cap\left|u^{n}\right| \leq k\right\}} k^{r-1} d x d t \\
& \quad+\int_{\left\{\left|\nabla u^{n}\right|^{\lambda}>k \cap\left|u^{n}\right|>k\right\}} k^{r-1} d x d t \\
& \leq \int_{\left\{\left|\nabla u^{n}\right|^{\lambda}>k \cap\left|u^{n}\right| \leq k\right\}} k^{r-1} d x d t+\int_{\left\{\left|u^{n}\right|>k\right\}} k^{r-1} d x d t \\
& \leq \int_{\left\{\left|u^{n}\right| \leq k\right\}} k^{r-1}\left(\frac{\left|\nabla u^{n}\right|^{\lambda}}{k}\right)^{2 / \lambda} d x d t \\
& \quad+\int_{Q_{T}}\left|u^{n}\right|^{r-1} d x d t \\
& \leq \frac{1}{k} \int_{\left\{\left\{\left|u^{n}\right| \leq k\right\}\right.}\left|\nabla u^{n}\right|^{2} d x d t+\int_{Q_{T}}\left|u^{n}\right|^{r-1} d x d t \\
& \leq \frac{1}{k} \int_{Q_{T}}\left|\nabla \varphi_{k}\left(u^{n}\right)\right|^{2} d x d t+\int_{Q_{T}}\left|u^{n}\right|^{r-1} d x d t .
\end{aligned}
$$


Similarly we can obtain

$$
\begin{aligned}
\int_{\left\{\Gamma\left(\left|\nabla u^{n}\right|^{\lambda}>k\right)\right\}} k^{r-1} d S d t \leq & \frac{1}{k} \int_{\Gamma_{T}}\left|\nabla_{\Gamma} \varphi_{k}\left(u^{n}\right)\right|^{2} d S d t \\
& +\int_{\Gamma_{T}}\left|u^{n}\right|^{r-1} d S d t .
\end{aligned}
$$

Combining (28) and (29), it yields

$$
\begin{aligned}
& \int_{\left\{\Omega\left(\left|\nabla u^{n}\right|^{\lambda}>k\right)\right\}} k^{r-1} d x d t+\int_{\left\{\Gamma\left(\left|\nabla u^{n}\right|^{\lambda}>k\right)\right\}} k^{r-1} d S d t \\
& \leq \frac{1}{k}\left(\int_{Q_{T}}\left|\nabla \varphi_{k}\left(u^{n}\right)\right|^{2} d x d t+\int_{\Gamma_{T}}\left|\nabla_{\Gamma} \varphi_{k}\left(u^{n}\right)\right|^{2} d S d t\right) \\
& \quad+\int_{Q_{T}}\left|u^{n}\right|^{r-1} d x d t+\int_{\Gamma_{T}}\left|u^{n}\right|^{r-1} d S d t \leq C_{6}+C_{7},
\end{aligned}
$$

which implies that $\left|\nabla u^{n}\right|^{\lambda}$ is bounded in $M^{r-1}\left(Q_{T}, d \nu\right)$. Hence, we conclude from Lemma 5 that $\left|\nabla u^{n}\right|^{\beta}$ is bounded in $L^{1}(0, T ;(\bar{\Omega}, d \nu))$ for $1<\beta<2(r-1) / r$, which implies that $\left|\nabla u^{n}\right|^{q_{0}}$ is bounded in $L^{1}(0, T ;(\bar{\Omega}, d \nu))$ for $1<q_{0}<2(r-1) / r$. Therefore, $u^{n}(t)$ is bounded in $L^{q_{0}}\left(0, T ; W^{1, q_{0}}(\bar{\Omega}, d \nu)\right)$.

Furthermore, we obtain that $\left\{u_{t}^{n}\right\}$ is bounded in $L^{q_{0}}\left(0, T ; W^{-1, q_{0}}(\bar{\Omega}, d \nu)\right)+L^{1}\left(0, T ; L^{1}(\bar{\Omega}, d \nu)\right)$. Let $X=$ $W^{1, q_{0}}(\bar{\Omega}, d \nu), Z=L^{1}(\bar{\Omega}, d \nu), Y=W^{-1, q_{0}}(\bar{\Omega}, d \nu)+L^{1}(\bar{\Omega}, d \nu)$; using Lemma 6 , we know that $u^{n}(t)$ is relatively compact in $L^{1}\left(0, T ; L^{1}(\bar{\Omega}, d \nu)\right)$. Thus, up to a subsequence $u^{n}$ convergence to $u$ in $L^{1}\left(0, T ; L^{1}(\bar{\Omega}, d \nu)\right)$.

Step 2. $f\left(u^{n}\right)$ converges to $f(u)$ in $L^{1}\left(0, T ;\left(L^{1}(\bar{\Omega}, d \nu)\right)\right.$ for any given $T>0$.

By Vitali's convergence theorem, it is enough to prove

$$
\begin{aligned}
& \lim _{|E| \rightarrow 0} \sup _{n} \int_{E}\left|f\left(u^{n}\right)\right| d x d t \\
& +\lim _{|E| \rightarrow 0} \sup _{n} \int_{E}\left|f\left(u^{n}\right)\right| d S d t=0 .
\end{aligned}
$$

Given $L>0$, we define

$$
T_{L}(s)= \begin{cases}\operatorname{sgn}(s) & |s|>L, \\ 0 & |s| \leq L .\end{cases}
$$

Let $\left\{T_{L}^{i}\right\}$ be a sequence of real smooth increasing functions with $T_{L}^{i}(0)=0$ and $T_{L}^{i}(s) \longrightarrow T_{L}(s)$ as $i \longrightarrow+\infty$. Taking $T_{L}^{i}\left(u^{n}\right)$ as a test function in (15), we deduce that for any $T>0$

$$
\begin{aligned}
& \int_{\Omega} \Theta_{L}^{i}\left(u^{n}(T)\right) d x+\int_{\Gamma} \Theta_{L}^{i}\left(u^{n}(T)\right) d S \\
& \quad+\int_{Q_{T}} f\left(u^{n}\right) T_{L}^{i}\left(u^{n}\right) d x d t \\
& \quad+\int_{\Gamma_{T}} f\left(u^{n}\right) T_{L}^{i}\left(u^{n}\right) d S d t \\
& \leq \int_{Q_{T}}\left|h^{n} T_{L}^{i}\left(u^{n}\right)\right| d x d t+\int_{\Gamma_{T}}\left|g^{n} T_{L}^{i}\left(u^{n}\right)\right| d S d t \\
& \quad+\int_{\Omega} \Theta_{L}^{i}\left(u_{0}^{n}\right) d x+\int_{\Gamma} \Theta_{L}^{i}\left(u_{0}^{n}\right) d S
\end{aligned}
$$

where $\Theta_{L}^{i}$ is the primitive function of $T_{L}^{i}$. Note that the first integral is nonnegative; thus discarding it and passing to the limit in $i$, we obtain that

$$
\begin{aligned}
& \int_{\left\{\Omega\left(\left|u^{n}\right|>L\right)\right\}} f\left(u^{n}\right) \operatorname{sgn}\left(u^{n}\right) d x d t \\
& \quad+\int_{\left\{\Gamma\left(\left|u^{n}\right|>L\right)\right\}} f\left(u^{n}\right) \operatorname{sgn}\left(u^{n}\right) d S d t \\
& \leq \int_{\left\{\left|u^{n}\right|>L\right\}}\left|h^{n}\right| d x d t+\int_{\left\{\left|u^{n}\right|>L\right\}}\left|g^{n}\right| d S d t \\
& \quad+\int_{\left\{\Omega\left(\left|u_{0}^{n}\right|>L\right)\right\}}\left|u_{0}^{n}\right| d x+\int_{\left\{\Gamma\left(\left|u_{0}^{n}\right|>L\right)\right\}}\left|u_{0}^{n}\right| d S .
\end{aligned}
$$

From (2), we know that when $|s| \geq \sigma_{0}$ we have

$$
c_{1}|s|^{r-1}-\frac{C}{\sigma_{0}} \leq f(s) \operatorname{sgn}(s) \leq c_{2}|s|^{r-1}+\frac{C}{\sigma_{0}},
$$

and when $|s| \leq \sigma_{0}$, we have $|f|<c_{6}$ Combining this with (35), we obtain that for any $s \in \mathbb{R}$

$$
\begin{gathered}
c_{1}|s|^{r-1}-\frac{C}{\sigma_{0}}-c_{6} \leq f(s) \operatorname{sgn}(s) \leq c_{2}|s|^{r-1}+\frac{C}{\sigma_{0}}+c_{6} \\
|f(s)| \leq C_{2}|s|^{r-1}+C_{1}+c_{6},
\end{gathered}
$$

where $C_{1}=C / \sigma_{0}, C_{2}=\max \left\{c_{1}, c_{2}\right\}$. This implies that

$$
|f(s)| \leq C_{3}(f(s) \operatorname{sgn}(s)+1)
$$

for some positive constant $C_{3}$. Thus, we deduce from (34) that

$$
\begin{aligned}
& \int_{\left\{\Omega\left(\left|u^{n}\right|>L\right)\right\}}\left|f\left(u^{n}\right)\right| d x d t+\int_{\left\{\Gamma\left(\left|u^{n}\right|>L\right)\right\}}\left|f\left(u^{n}\right)\right| d S d t \\
& \leq C_{3}\left(\int_{\left\{\left|u^{n}\right|>L\right\}}\left|h^{n}\right| d x d t+\int_{\left\{\left|u^{n}\right|>L\right\}}\left|g^{n}\right| d S d t\right. \\
& +\int_{\left\{\Omega\left(\left|u_{0}^{n}\right|>L\right)\right\}}\left|u_{0}^{n}\right| d x+\int_{\left\{\Gamma\left(\left|u_{0}^{n}\right|>L\right)\right\}}\left|u_{0}^{n}\right| d S \\
& \left.+\int_{\left\{\Omega\left(\left|u_{0}^{n}\right|>L\right)\right\}} d x d t+\int_{\left\{\Gamma\left(\left|u_{0}^{n}\right|>L\right)\right\}} d S d t\right) .
\end{aligned}
$$

Since for any $T>0, u^{n}$ converges to $u$ in $L^{1}(0, T ;(\bar{\Omega}, d \nu))$; there exists a positive constant $C_{4}$ independent of $n$, such that

$$
\begin{gathered}
\int_{\left\{\Omega\left(\left|u^{n}\right|>L\right)\right\}}\left|u^{n}\right| d x d t+\int_{\left\{\Gamma\left(\left|u^{n}\right|>L\right)\right\}}\left|u^{n}\right| d S d t \\
\leq \int_{Q_{T}}\left|u^{n}\right| d x d t+\int_{\Gamma_{T}}\left|u^{n}\right| d S d t<C_{4},
\end{gathered}
$$

for any $L \geq 0$.

Then we have

$$
\begin{aligned}
& \left|\left\{\Omega\left(\left|u^{n}\right|>L\right)\right\}\right|+\left|\left\{\Gamma\left(\left|u^{n}\right|>L\right)\right\}\right| \\
& \quad=\operatorname{meas}\left\{\Omega\left(\left|u^{n}\right|>L\right)\right\}+\text { meas }\left\{\Gamma\left(\left|u^{n}\right|>L\right)\right\} \\
& \quad \leq \frac{1}{L}\left\|u^{n}\right\|_{L^{1}(\bar{\Omega}, d v)} \leq \frac{C_{4}}{L} .
\end{aligned}
$$


For any $\varepsilon>0$, we can always find a positive constant $L_{1}$ such that

$$
\begin{aligned}
& \left|\left\{\Omega\left(\left|u^{n}\right|>L\right)\right\}\right|+\left|\left\{\Gamma\left(\left|u^{n}\right|>L\right)\right\}\right|<\varepsilon, \\
& \text { holds for all } n \text {. }
\end{aligned}
$$

Now we analyze each term of the right hand side of (38); note that $\left\{h^{n}\right\}$ converges to $h$ in $L^{1}(\Omega)$, and

$$
\begin{aligned}
\int_{\left\{\Omega\left(\left|u^{n}\right|>L\right)\right\}}\left|h^{n}\right| d x d t \leq & \int_{\left\{\Omega\left(\left|u^{n}\right|>L\right)\right\}}\left|h^{n}-h\right| d x d t \\
& +\int_{\left\{\Omega\left(\left|u^{n}\right|>L\right)\right\}}|h| d x d t .
\end{aligned}
$$

For any given $\varepsilon>0$, the first term on the right hand side can be strictly less then $\varepsilon$ whenever $n>N_{1}$. Thanks to (41), for $L$ large enough $\left(L>L_{1}\right)$, the second term can be strictly less than $\varepsilon$ for all $n$ (absolute continuity of the Lebesgue integral). Also, we can always find a positive constant $L_{2}$, such that

$$
\int_{\left\{\Omega\left(\left|u^{n}\right|>L_{3}\right)\right\}}\left|h^{n}\right| d x d t<\varepsilon, \quad \text { for } n=1,2, \cdots N_{1} \text {. }
$$

So setting $L_{3}=\max \left\{L_{1}, L_{2}\right\}$ ), we get

$$
\int_{\left\{\Omega\left(\left|u^{n}\right|>L_{3}\right)\right\}}\left|h^{n}\right| d x d t<2 \varepsilon, \quad \text { for any } n .
$$

Similarly, setting $\left.L_{4}=\max \left\{L_{1}, L_{2}, L_{3}\right\}\right)$, we can get

$$
\int_{\left\{\Gamma\left(\left|u^{n}\right|>L_{4}\right)\right\}}\left|g^{n}\right| d S d t<2 \varepsilon, \quad \text { for any } n .
$$

Then for any $\varepsilon>0$ there exists a $L_{5}>0$, such that

$$
\int_{\left\{\Omega\left(\left|u_{0}^{n}\right|>L_{5}\right)\right\}}\left|u_{0}^{n}\right| d x+\int_{\left\{\Gamma\left(\left|u_{0}^{n}\right|>L_{5}\right)\right\}}\left|u_{0}^{n}\right| d S<2 \varepsilon
$$

for any $n$.

Taking (41)-(46) into (38), we obtain that for any $\varepsilon>0$ there exists $L_{6}=\max \left\{L_{1}, L_{3}, L_{4}, L_{5}\right\}$, such that

$$
\begin{aligned}
& \int_{E \cap\left\{\Omega\left(\left|u^{n}\right|>L^{6}\right)\right\}}\left|f\left(u^{n}\right)\right| d x d t \\
& \quad+\int_{E \cap\left\{\Gamma\left(\left|u^{n}\right|>L^{6}\right)\right\}}\left|f\left(u^{n}\right)\right| d S d t<\varepsilon
\end{aligned}
$$

uniformly in $n$. On the other hand, from (36) we have

$$
\begin{aligned}
& \int_{E \cap\left\{\Omega\left(\left|u^{n}\right|>L^{6}\right)\right\}}\left|f\left(u^{n}\right)\right| d x d t \\
& +\int_{E \cap\left\{\Gamma\left(\left|u^{n}\right| \leq L^{6}\right)\right\}}\left|f\left(u^{n}\right)\right| d S d t \\
& \quad \leq \int_{E}\left(C_{2}\left|L_{6}\right|^{r-1}+C_{2}+c_{4}\right)<\varepsilon
\end{aligned}
$$

uniformly in $n$, whenever $|E|$ is small enough.
Note that for any $E \subset\left(Q_{T}, d v\right)$

$$
\begin{aligned}
\int_{E}\left|f\left(u^{n}\right)\right|= & \int_{E \cap\left\{\Omega\left(\left|u^{n}\right|>L^{6}\right)\right\}}\left|f\left(u^{n}\right)\right| d x d t \\
& +\int_{E \cap\left\{\Omega\left(\left|u^{n}\right| \leq L^{6}\right)\right\}}\left|f\left(u^{n}\right)\right| d S d t<\varepsilon .
\end{aligned}
$$

Thus, for any $T>0, f\left(u^{n}\right)$ is equi-integrable in $\left(Q_{T}, d \nu\right)$ and due to Vitali's convergence theorem, $f\left(u^{n}\right)$ converges to $f(u)$ in $L^{1}\left(0, T ; L^{1}(\bar{\Omega}, d \nu)\right)$.

Step 3. $\nabla u^{n}$ converges to a function $\nabla u$ in $L^{1}\left(0, T ; L^{1}(\bar{\Omega}, d \nu)\right)$.

For $\varepsilon>0$, taking $\phi=\varphi_{\varepsilon}\left(u^{n}-u^{m}\right)$ as a test function in (15) one can deduce that

$$
\begin{aligned}
& \int_{\Omega} \Psi_{\varepsilon}\left(u^{n}-u^{m}\right)(t) d x+\int_{\Gamma} \Psi_{\varepsilon}\left(u^{n}-u^{m}\right)(t) d S \\
& \quad+\int_{Q_{T}}\left|\nabla u^{n}-\nabla u^{m}\right|^{2} \varphi_{\varepsilon}^{\prime}\left(u^{n}-u^{m}\right) d x d t \\
& \quad+\int_{\Gamma_{T}}\left|\nabla_{\Gamma} u^{n}-\nabla_{\Gamma} u^{m}\right|^{2} \varphi_{\varepsilon}^{\prime}\left(u^{n}-u^{m}\right) d S d t \\
& \leq \int_{Q_{T}}\left|\left(f\left(u^{n}\right)-f\left(u^{m}\right)\right) \varphi_{\varepsilon}\left(u^{n}-u^{m}\right)\right| d x d t \\
& \quad+\int_{\Gamma_{T}}\left|\left(f\left(u^{n}\right)-f\left(u^{m}\right)\right) \varphi_{\varepsilon}\left(u^{n}-u^{m}\right)\right| d S d t \\
& \quad+\int_{Q_{T}}\left|\left(h^{n}-h^{m}\right) \varphi_{\varepsilon}\left(u^{n}-u^{m}\right)\right| d x d t \\
& \quad+\int_{\Gamma_{T}}\left(g^{n}-g^{m}\right) \varphi_{\varepsilon}\left(u^{n}-u^{m}\right) d S d t \\
& \quad+\int_{\Omega} \Psi_{\varepsilon}\left(u^{n}-u^{m}\right)(0) d x \\
& \quad+\int_{\Gamma} \Psi_{\varepsilon}\left(u^{n}-u^{m}\right)(0) d S .
\end{aligned}
$$

Note that $f\left(u^{n}\right), h^{n}, g^{n}$, and $u_{0}^{n}$ are convergent in $L^{1}$, and $0 \leq$ $\Psi_{\varepsilon}(s) \leq \varepsilon$, for $0<\varepsilon<1$ we obtain that

$$
\begin{aligned}
& \int_{Q_{T}}\left|\nabla u^{n}-\nabla u^{m}\right|^{2} \varphi_{\varepsilon}^{\prime}\left(u^{n}-u^{m}\right) d x d t \\
& \quad+\int_{\Gamma_{T}}\left|\nabla_{\Gamma} u^{n}-\nabla_{\Gamma} u^{m}\right|^{2} \varphi_{\varepsilon}^{\prime}\left(u^{n}-u^{m}\right) d S d t \\
& \quad=\int_{Q_{T}^{1}}\left|\nabla u^{n}-\nabla u^{m}\right|^{2} d x d t \\
& \quad+\int_{\Gamma_{T}^{1}}\left|\nabla_{\Gamma} u^{n}-\nabla_{\Gamma} u^{m}\right|^{2} d S d t \leq 2 c_{3} \varepsilon+2 c_{4} \varepsilon+2 c_{5} \varepsilon \\
& \quad+2 c_{6} \varepsilon<C \varepsilon,
\end{aligned}
$$

where

$$
\begin{aligned}
& Q_{T}^{1}=\left\{(x, t) \in Q_{T}:\left|u^{n}-u^{m}\right| \leq \varepsilon\right\}, \\
& \Gamma_{T}^{1}=\left\{(x, t) \in \Gamma_{T}:\left|u^{n}-u^{m}\right| \leq \varepsilon\right\} .
\end{aligned}
$$


Using Hölder inequality, we get

$$
\begin{aligned}
& \int_{Q_{T}}\left|\nabla u^{n}-\nabla u^{m}\right| d x d t \\
& =\int_{Q_{T}^{1}}\left|\nabla u^{n}-\nabla u^{m}\right| d x d t \\
& \quad+\int_{Q_{T} \backslash Q_{T}^{1}}\left|\nabla u^{n}-\nabla u^{m}\right| d x d t \\
& \leq c\left(\int_{Q_{T}^{1}}\left|\nabla u^{n}-\nabla u^{m}\right|^{2} d x d t\right)^{1 / 2} \\
& \quad+C m e a s\left\{(x, t):\left|u^{n}-u^{m}\right|>\varepsilon\right\} \\
& \leq C \varepsilon^{1 / 2}+\text { Cmeas }\left(A_{1}\right),
\end{aligned}
$$

where $A_{1}=Q_{T} \backslash Q_{T}^{1}=\left\{(x, t) \in Q_{T}:\left|u^{n}-u^{m}\right|>\varepsilon\right\}$. Now let us bound meas $\left(A_{1}\right)$; we have

$$
\begin{aligned}
\int_{\mathrm{Q}_{T}}\left|u^{n}-u^{m}\right| d x d t & \geq \int_{A_{1}}\left|u^{n}-u^{m}\right| d x d t \\
& \geq \operatorname{cmeas}\left(A_{1}\right)
\end{aligned}
$$

$u^{n} \longrightarrow u$ in $L^{1}(0, T ;(\bar{\Omega}, d \nu))$; hence, we have meas $\left(A_{1}\right)<\varepsilon$. Then we have

$$
\int_{Q_{T}}\left|\nabla u^{n}-\nabla u^{m}\right| d x d t<\varepsilon
$$

In the same way, we have $\int_{\Gamma_{T}}\left|\nabla_{\Gamma} u^{n}-\nabla_{\Gamma} u^{m}\right| d S d t<\varepsilon$. So we can obtain that

$$
\left|\nabla u^{n}-\nabla u^{m}\right|_{L^{1}\left(0, T ; L^{1}(\bar{\Omega}, d v)\right)}<\varepsilon
$$

That is $\left\{\nabla u^{n}\right\}$ is a Cauchy sequence in $L^{1}\left(0, T ; L^{1}(\bar{\Omega}, d \nu)\right)$. Discarding the nonnegative term in the left hand side of (50) we can deduce that $u^{n} \longrightarrow u$ in $C\left(0, T ; L^{1}(\bar{\Omega}, d v)\right)$.

Step 4. Pass to the limits.

Similar to [29], taking $\varphi_{k}\left(u^{n}-\phi\right)$ as a test function in (15), we have

$$
\begin{aligned}
\int_{\Omega} \Psi_{k} & \left(u^{n}-\phi\right)(T) d x-\int_{\Omega} \Psi_{k}\left(u^{n}-\phi\right)(0) d x \\
& +\int_{\Gamma} \Psi_{k}\left(u^{n}-\phi\right)(T) d S d t \\
& +\int_{\Gamma} \Psi_{k}\left(u^{n}-\phi\right)(0) d S \\
& +\int_{Q_{T}} \phi_{t} \varphi_{k}\left(u^{n}-\phi\right) d x d t \\
& +\int_{\Gamma_{T}} \phi_{t} \varphi_{k}\left(u^{n}-\phi\right) d S d t \\
& +\int_{Q_{T}} f\left(u^{n}\right) \varphi_{k}\left(u^{n}-\phi\right) d x d t
\end{aligned}
$$

$$
\begin{aligned}
& +\int_{\Gamma_{T}} f\left(u^{n}\right) \varphi_{k}\left(u^{n}-\phi\right) d S d t \\
& +\int_{Q_{T}} \nabla u^{n} \nabla \varphi_{k}\left(u^{n}-\phi\right) d x d t \\
& +\int_{\Gamma_{T}} \nabla_{\Gamma} u^{n} \nabla_{\Gamma} \varphi_{k}\left(u^{n}-\phi\right) d S d t \\
& =\int_{Q_{T}} h^{n} \varphi_{k}\left(u^{n}-\phi\right) d x d t \\
& +\int_{\Gamma_{T}} g^{n} \varphi_{k}\left(u^{n}-\phi\right) d S d t,
\end{aligned}
$$

where $\phi \in L^{2}\left(0, T ; w^{1,2}(\bar{\Omega}, d \nu)\right) \cap L^{\infty}\left(0, T ; L^{\infty}(\bar{\Omega}, d \nu)\right)$. Let us study the limit for $n \longrightarrow \infty$ of each term.

We have seen that $u^{n} \longrightarrow u$ in $C\left(0, T ; L^{1}(\bar{\Omega}, d \nu)\right)$; hence, $\forall t \leq T, u^{n} \longrightarrow u$ in $L^{1}(\bar{\Omega}, d \nu)$. Since $\Psi_{k}$ is k-Lipschitz continuous one has, when $n \longrightarrow \infty$

$$
\begin{aligned}
\int_{\Omega} \Psi_{k}\left(u^{n}-\phi\right)(T) d x+\int_{\Gamma} \Psi_{k}\left(u^{n}-\phi\right)(T) d S \\
\quad \longrightarrow \int_{\Omega} \Psi_{k}(u-\phi)(T) d x+\int_{\Gamma} \Psi_{k}(u-\phi)(T) d S
\end{aligned}
$$

Since

$$
\begin{aligned}
& \int_{\Omega} \Psi_{k}\left(u^{n}-\phi\right)(0) d x+\int_{\Gamma} \Psi_{k}\left(u^{n}-\phi\right)(0) d S \\
& =\int_{\Omega} \Psi_{k}\left(u_{0}^{n}-\phi(0)\right) d x+\int_{\Gamma} \Psi_{k}\left(u_{0}^{n}-\phi(0)\right) d S,
\end{aligned}
$$

and $u_{0}^{n} \longrightarrow u_{0}$ in $L^{1}(\Omega)$, one has similarly

$$
\begin{aligned}
& \int_{\Omega} \Psi_{k}\left(u^{n}-\phi\right)(0) d x+\int_{\Gamma} \Psi_{k}\left(u^{n}-\phi\right)(0) d S \\
& \longrightarrow \int_{\Omega} \Psi_{k}\left(u_{0}-\phi(0)\right) d x+\int_{\Gamma} \Psi_{k}\left(u_{0}-\phi(0)\right) d S .
\end{aligned}
$$

We now pass to the limit in $\int_{Q_{T}} \phi_{t} \varphi_{k}\left(u^{n}-\phi\right) d x d t+$ $\int_{\Gamma_{T}} \phi_{t} \varphi_{k}\left(u^{n}-\phi\right) d S d t$. Using the hypothesis $\phi \in$ $L^{\infty}\left(0, T ; L^{\infty}(\bar{\Omega}, d \nu)\right), \varphi_{k}\left(u^{n}-\phi\right) \in L^{2}\left(0, T ; H^{1}(\bar{\Omega}, d \nu)\right)$. Since $\varphi_{k}\left(u^{n}-\phi\right) \longrightarrow \varphi_{k}(u-\phi)$ weakly in $L^{2}\left(0, T ; H^{1}(\Omega, d \nu)\right)$, we have

$$
\begin{gathered}
\int_{Q_{T}} \phi_{t} \varphi_{k}\left(u^{n}-\phi\right) d x d t+\int_{\Gamma_{T}} \phi_{t} \varphi_{k}\left(u^{n}-\phi\right) d S d t \\
\longrightarrow \int_{Q_{T}} \phi_{t} \varphi_{k}(u-\phi) d x d t \\
\quad+\int_{\Gamma_{T}} \phi_{t} \varphi_{k}(u-\phi) d S d t
\end{gathered}
$$


Moreover,

$$
\begin{aligned}
\int_{Q_{T}} \nabla & u^{n} \nabla \varphi_{k}\left(u^{n}-\phi\right) d x d t \\
= & \int_{Q_{T}} \nabla\left(u^{n}-\phi\right) \nabla \varphi_{k}\left(u^{n}-\phi\right) d x d t \\
& +\int_{Q_{T}} \nabla \phi \nabla \varphi_{k}\left(u^{n}-\phi\right) d x d t \\
= & \int_{Q_{T}}\left|\nabla\left(u^{n}-\phi\right)\right|^{2} \varphi_{k}^{\prime}\left(u^{n}-\phi\right) d x d t \\
& +\int_{Q_{T}} \nabla \phi \nabla \varphi_{k}\left(u^{n}-\phi\right) d x d t \\
= & \int_{Q_{T}}\left|\nabla \varphi_{k}\left(u^{n}-\phi\right)\right|^{2} d x d t \\
& +\int_{Q_{T}} \nabla \phi \nabla \varphi_{k}\left(u^{n}-\phi\right) d x d t .
\end{aligned}
$$

Since $\nabla \varphi_{k}\left(u^{n}-\phi\right) \quad \longrightarrow \quad \nabla \varphi_{k}(u-\phi)$ weakly in $L^{2}\left(0, T ; L^{2}(\bar{\Omega}, d \nu)\right)$, we have

$$
\begin{aligned}
& \int_{Q_{T}} \nabla \phi \nabla \varphi_{k}\left(u^{n}-\phi\right) d x d t \\
& \quad \longrightarrow \int_{Q_{T}} \nabla \phi \nabla \varphi_{k}(u-\phi) d x d t
\end{aligned}
$$

It follows from Fatou's lemma that

$$
\begin{aligned}
& \int_{Q_{T}}\left|\nabla \varphi_{k}(u-\phi)\right|^{2} d x d t \\
& \leq \lim _{n \rightarrow \infty} \int_{Q_{T}}\left|\nabla \varphi_{k}\left(u^{n}-\phi\right)\right|^{2} d x d t .
\end{aligned}
$$

Combining (62)-(64) we have

$$
\begin{aligned}
\lim _{n \rightarrow \infty} & \int_{Q_{T}} \nabla u^{n} \nabla \varphi_{k}\left(u^{n}-\phi\right) d x d t \\
\geq & \int_{Q_{T}^{1}}\left|\nabla \varphi_{k}(u-\phi)\right|^{2} d x d t \\
& \quad+\int_{Q_{T}^{1}} \nabla \phi \nabla \varphi_{k}(u-\phi) d x d t \\
= & \int_{Q_{T}^{1}}\left(\nabla \varphi_{k}(u-\phi)\right)\left(\nabla \varphi_{k}(u-\phi)+\nabla \phi\right) d x d t \\
= & \int_{Q_{T}}(\nabla u-\nabla \phi+\nabla \phi) \nabla \varphi_{k}(u-\phi) d x d t \\
= & \int_{Q_{T}} \nabla u \nabla \varphi_{k}(u-\phi) d x d t .
\end{aligned}
$$

Similarly, we can obtain

$$
\begin{gathered}
\lim _{n \rightarrow \infty} \int_{\Gamma_{T}} \nabla_{\Gamma} u^{n} \nabla_{\Gamma} \varphi_{k}\left(u^{n}-\phi\right) d S d t \\
\quad \geq \int_{\Gamma_{T}} \nabla_{\Gamma} u \nabla_{\Gamma} \varphi_{k}(u-\phi) d S d t
\end{gathered}
$$

Finally since $\varphi_{k}\left(u^{n}-\phi\right)$ converges to $\varphi_{k}(u-$ $\phi)$ weakly ${ }^{*} L^{\infty}\left(0, T ; L^{\infty}(\bar{\Omega}, d \nu)\right)$ and $f\left(u^{n}\right) \longrightarrow f(u)$ in $L^{1}(0, T ;(\bar{\Omega}, d \nu)), h^{n} \longrightarrow h$ in $L^{1}\left(Q_{T}\right), g^{n} \longrightarrow g$ in $L^{1}\left(\Gamma_{T}\right)$, we obtain that

$$
\begin{aligned}
& \int_{Q_{T}} f\left(u^{n}\right) \varphi_{k}\left(u^{n}-\phi\right) d x d t \\
& \quad+\int_{\Gamma_{T}} f\left(u^{n}\right) \varphi_{k}\left(u^{n}-\phi\right) d S d t \\
& \quad \longrightarrow \int_{Q_{T}} f(u) \varphi_{k}(u-\phi) d x d t \\
& \quad+\int_{\Gamma_{T}} f(u) \varphi_{k}(u-\phi) d S d t, \\
& \int_{Q_{T}} h^{n} \varphi_{k}\left(u^{n}-\phi\right) d x d t \longrightarrow \int_{Q_{T}} h \varphi_{k}(u-\phi) d x d t, \\
& \int_{\Gamma_{T}} g^{n} \varphi_{k}\left(u^{n}-\phi\right) d S d t \longrightarrow \int_{\Gamma_{T}} g \varphi_{k}(u-\phi) d S d t .
\end{aligned}
$$

Therefore passing to the limit in (57), it yields

$$
\begin{aligned}
& \int_{\Omega} \Psi_{k}(u-\phi)(T) d x-\int_{\Omega} \Psi_{k}(u-\phi)(0) d x \\
& \quad+\int_{\Gamma} \Psi_{k}(u-\phi)(T) d S-\int_{\Gamma} \Psi_{k}(u-\phi)(0) d S \\
& \quad+\int_{Q_{T}} \phi_{t} \varphi_{k}(u-\phi) d x d t \\
& \quad+\int_{\Gamma_{T}} \phi_{t} \varphi_{k}(u-\phi) d S d t \\
& \quad+\int_{Q_{T}} f(u) \varphi_{k}(u-\phi) d x d t \\
& \quad+\int_{\Gamma_{T}} f(u) \varphi_{k}(u-\phi) d S d t \\
& \quad+\int_{Q_{T}} \nabla u \nabla \varphi_{k}(u-\phi) d x d t \\
& \quad+\int_{\Gamma_{T}} \nabla_{\Gamma} u \nabla_{\Gamma} \varphi_{k}(u-\phi) d S d t \\
& \leq \int_{Q_{T}} h \varphi_{k}(u-\phi) d x d t+\int_{\Gamma_{T}} g \varphi_{k}(u-\phi) d S d t .
\end{aligned}
$$

Step 5. Uniqueness of entropy solutions

Assume that there is another entropy solution $\bar{u}$ to the problem. For any $T>0$, taking $\phi=u^{n}$ as a test function we have

$$
\begin{aligned}
& \int_{\Omega} \Psi_{k}\left(\bar{u}-u^{n}\right)(T) d x-\int_{\Omega} \Psi_{k}\left(\bar{u}-u^{n}\right)(0) d x \\
& \quad+\int_{\Gamma} \Psi_{k}\left(\bar{u}-u^{n}\right)(T) d S-\int_{\Gamma} \Psi_{k}\left(\bar{u}-u^{n}\right)(0) d S
\end{aligned}
$$




$$
\begin{aligned}
& +\int_{Q_{T}} u_{t}^{n} \varphi_{k}\left(\bar{u}-u^{n}\right) d x d t \\
& +\int_{\Gamma_{T}} u_{t}^{n} \varphi_{k}(\bar{u}-\phi) d S d t \\
& +\int_{Q_{T}} f(\bar{u}) \varphi_{k}\left(\bar{u}-u^{n}\right) d x d t \\
& +\int_{\Gamma_{T}} f(\bar{u}) \varphi_{k}\left(\bar{u}-u^{n}\right) d S d t \\
& +\int_{Q_{T}} \nabla \bar{u} \nabla \varphi_{k}\left(\bar{u}-u^{n}\right) d x d t \\
& +\int_{\Gamma_{T}} \nabla_{\Gamma} \bar{u} \nabla_{\Gamma} \varphi_{k}\left(\bar{u}-u^{n}\right) d S d t \\
& \leq \int_{Q_{T}} h \varphi_{k}\left(\bar{u}-u^{n}\right) d x d t+\int_{\Gamma_{T}} g \varphi_{k}\left(\bar{u}-u^{n}\right) d S d t
\end{aligned}
$$

and taking $\varphi_{k}\left(\bar{u}-u^{n}\right)$ as a test function in (15), we obtain that

$$
\begin{aligned}
& \int_{Q_{T}} u_{t}^{n} \varphi_{k}\left(\bar{u}-u^{n}\right) d x d t+\int_{\Gamma_{T}} u_{t}^{n} \varphi_{k}(\bar{u}-\phi) d S d t \\
& \quad+\int_{Q_{T}} f\left(u^{n}\right) \varphi_{k}\left(\bar{u}-u^{n}\right) d x d t \\
& \quad+\int_{\Gamma_{T}} f\left(u^{n}\right) \varphi_{k}\left(\bar{u}-u^{n}\right) d S d t \\
& \quad+\int_{Q_{T}} \nabla u^{n} \nabla \varphi_{k}\left(\bar{u}-u^{n}\right) d x d t \\
& \quad+\int_{\Gamma_{T}} \nabla_{\Gamma} u^{n} \nabla_{\Gamma} \varphi_{k}\left(\bar{u}-u^{n}\right) d S d t \\
& =\int_{Q_{T}} h^{n} \varphi_{k}\left(\bar{u}-u^{n}\right) d x d t \\
& \quad+\int_{\Gamma_{T}} g^{n} \varphi_{k}\left(\bar{u}-u^{n}\right) d S d t .
\end{aligned}
$$

Subtracting (70) from (69), it yields

$$
\begin{aligned}
& \int_{\Omega} \Psi_{k}\left(\bar{u}-u^{n}\right)(T) d x-\int_{\Omega} \Psi_{k}\left(\bar{u}-u^{n}\right)(0) d x \\
& \quad+\int_{\Gamma} \Psi_{k}\left(\bar{u}-u^{n}\right)(T) d S-\int_{\Gamma} \Psi_{k}\left(\bar{u}-u^{n}\right)(0) d S \\
& \quad+\int_{Q_{T}} f\left(\bar{u}-u^{n}\right) \varphi_{k}\left(\bar{u}-u^{n}\right) d x d t \\
& \quad+\int_{\Gamma_{T}} f\left(\bar{u}-u^{n}\right) \varphi_{k}\left(\bar{u}-u^{n}\right) d S d t \\
& \quad+\int_{Q_{T}} \nabla\left(\bar{u}-\nabla u^{n}\right) \nabla \varphi_{k}\left(\bar{u}-u^{n}\right) d x d t \\
& \quad+\int_{\Gamma_{T}}\left(\nabla_{\Gamma} \bar{u}-\nabla_{\Gamma} u^{n}\right) \nabla_{\Gamma} \varphi_{k}\left(\bar{u}-u^{n}\right) d S d t
\end{aligned}
$$

$$
\begin{aligned}
& \leq \int_{Q_{T}}\left(h-h^{n}\right) \varphi_{k}\left(\bar{u}-u^{n}\right) d x d t \\
& \quad+\int_{\Gamma_{T}}\left(g-g^{n}\right) \varphi_{k}\left(\bar{u}-u^{n}\right) d S d t .
\end{aligned}
$$

Note that

$$
\begin{aligned}
& \Psi_{k}\left(\bar{u}-u^{n}\right)(T) \leq k\left(|\bar{u}(T)|+\left|u^{n}(T)\right|\right), \\
& \Psi_{k}\left(\bar{u}-u^{n}\right)(0) \leq k\left(|\bar{u}(0)|+\left|u^{n}(0)\right|\right) .
\end{aligned}
$$

Letting $n \longrightarrow \infty$, we have

$$
\begin{aligned}
& \int_{\Omega} \Psi_{k}\left(\bar{u}-u^{n}\right)(T) d x+\int_{\Gamma} \Psi_{k}\left(\bar{u}-u^{n}\right)(T) d S \\
& \longrightarrow \int_{\Omega} \Psi_{k}(\bar{u}-u)(T) d x+\int_{\Gamma} \Psi_{k}(\bar{u}-u)(T) d S, \\
& \int_{\Omega} \Psi_{k}\left(\bar{u}-u^{n}\right)(0) d x+\int_{\Gamma} \Psi_{k}\left(\bar{u}-u^{n}\right)(0) d S \longrightarrow 0,
\end{aligned}
$$

On the other hand, since $h^{n} \longrightarrow h$ in $L^{1}(\Omega), g^{n} \longrightarrow$ $g$ in $L^{1}(\Gamma)$, we obtain

$$
\begin{aligned}
& \int_{Q_{T}}\left(h-h^{n}\right) \varphi_{k}\left(\bar{u}-u^{n}\right) d x d t \rightarrow 0, \\
& \int_{\Gamma_{T}}\left(g-g^{n}\right) \varphi_{k}\left(\bar{u}-u^{n}\right) d S d t \longrightarrow 0 .
\end{aligned}
$$

Thanks to the assumption on $f$, we have

$$
\begin{aligned}
& -\int_{Q_{T}} f\left(\bar{u}-u^{n}\right) \varphi_{k}\left(\bar{u}-u^{n}\right) d x d t \\
& \quad \leq \int_{Q_{T}} l\left(\bar{u}-u^{n}\right) \varphi_{k}\left(\bar{u}-u^{n}\right) d x d t \\
& \quad \leq 2 l \int_{Q_{T}} \Psi_{k}\left(\bar{u}-u^{n}\right) d x d t, \\
& -\int_{\Gamma_{T}} f\left(\bar{u}-u^{n}\right) \varphi_{k}\left(\bar{u}-u^{n}\right) d S d t \\
& \quad \leq \int_{\Gamma_{T}} l\left(\bar{u}-u^{n}\right) \varphi_{k}\left(\bar{u}-u^{n}\right) d S d t \\
& \quad \leq 2 l \int_{\Gamma_{T}} \Psi_{k}\left(\bar{u}-u^{n}\right) d S d t .
\end{aligned}
$$

Since

$$
\begin{aligned}
& \int_{Q_{T}}\left(\nabla \bar{u}-\nabla u^{n}\right) \nabla \varphi_{k}\left(\bar{u}-u^{n}\right) d x d t \\
& =\int_{Q_{T}}\left|\nabla \bar{u}-\nabla u^{n}\right|^{2} \varphi^{\prime}\left(\bar{u}-u^{n}\right) d x d t \geq 0, \\
& \int_{\Gamma_{T}}\left(\nabla_{\Gamma} \bar{u}-\nabla_{\Gamma} u^{n}\right) \nabla_{\Gamma} \varphi_{k}\left(\bar{u}-u^{n}\right) d S d t \\
& =\int_{\Gamma_{T}}\left|\nabla_{\Gamma} \bar{u}-\nabla_{\Gamma} u^{n}\right|^{2} \varphi^{\prime}\left(\bar{u}-u^{n}\right) d S d t \geq 0 .
\end{aligned}
$$


Omitting them and taking $n \longrightarrow \infty$ in $(71)$, we obtain

$$
\begin{aligned}
& \frac{d}{d t}\left(\int_{\Omega} \Psi_{k}(\bar{u}-u)(t) d x+\int_{\Gamma} \Psi_{k}(\bar{u}-u)(t) d S\right) \\
& \quad \leq 2 l\left(\int_{\Omega} \Psi_{k}(\bar{u}-u)(t) d x+\int_{\Gamma} \Psi_{k}(\bar{u}-u)(t) d S\right) .
\end{aligned}
$$

Then Gronwall's inequality implies that $\Psi_{k}(\bar{u}-u)(t)=0$ for all $t \in[0, T]$, and hence $\bar{u} \equiv u$.

\section{Existence of Global Attractors}

This section is devoted to the proof on the existence of the global attractor for the solution semigroup $\{S(t)\}_{t \geq 0}$.

Proof of Theorem 4 . We first prove continuity of the semigroup $\{S(t)\}_{t \geq 0}$ in $L^{1}(\bar{\Omega}, d \nu)$. Let $\{S(t)\}_{t \geq 0}$ be a family of operators corresponding to the entropy solution of problem (1) with

$$
S(t):\left(u_{0}, \gamma\left(u_{0}\right)\right) \longrightarrow(u(t), \gamma(u(t)))
$$

Then for any $t>0$, the map $S(t)$ is continuous map from $L^{1}(\bar{\Omega}, d \nu)$ to $L^{1}(\bar{\Omega}, d \nu)$.

The proof is similar to [32]. However, for the sack of completeness, we provide the details here. Let $u_{1}(t)$ and $u_{2}(t)$ be two solutions of problem (1) with initial data $u_{1,0}, u_{2,0}$, respectively. Assume that $u_{1}^{n}(t)$ and $u_{2}^{n}(t)$ are the solution of the approximate problem with initial data $u_{1,0}^{n}, u_{2,0}^{n}, h_{1}^{n}=h_{2}^{n}$ and $g_{1}^{n}=g_{2}^{n}$, respectively, such that for any $T>0$ and $\varepsilon>0$, there exists a $n_{0}$ such that for any $t \in[0, T]$

$$
\left\|u_{i}(t)-u_{i}^{n}(t)\right\|_{L^{1}(\bar{\Omega}, d v)}<\varepsilon, \quad \text { for } i=1,2 \text {, as } n \geq n_{0} .
$$

Here we may suppose that we have already extracted from $u_{i}^{n}$ a proper subsequence (the one converges to $u_{i}$ in $\left.C\left([0, T] ; L^{1}(\bar{\Omega}, d \nu)\right)\right)$, which is still denoted by $u_{i}^{n}, i=1,2$.

Note that $u_{1}^{n}-u_{2}^{n}$ satisfies the following equation:

$$
\begin{aligned}
& \left(u_{1}^{n}-u_{2}^{n}\right)_{t}-\Delta\left(u_{1}^{n}-u_{2}^{n}\right)+f\left(u_{1}^{n}\right)-f\left(u_{2}^{n}\right)=0 \\
& \quad \text { in } \Omega \times \mathbb{R}^{+}, \\
& \left(u_{1}^{n}-u_{2}^{n}\right)_{t}+\frac{\partial\left(u_{1}^{n}-u_{2}^{n}\right)}{\partial \nu}-\Delta_{\Gamma}\left(u_{1}^{n}-u_{2}^{n}\right)+f\left(u_{1}^{n}\right) \\
& -f\left(u_{2}^{n}\right)=0 \quad \text { on } \Gamma \times \mathbb{R}^{+}, \\
& u^{n}(x, 0)=u_{1,0}^{n}-u_{2,0}^{n} \quad \text { in } \Omega .
\end{aligned}
$$

Taking $(1 / k) \varphi_{k}\left(u_{1}^{n}-u_{2}^{n}\right)$ as a test function, and using the fact that

$$
\begin{gathered}
-\frac{1}{k} \int_{\Omega}\left(f\left(u_{1}^{n}\right)-f\left(u_{2}^{n}\right)\right) \varphi_{k}\left(u_{1}^{n}-u_{2}^{n}\right) d x \\
\quad \leq \frac{l}{k} \int_{\Omega}\left|u_{1}^{n}-u_{2}^{n}\right|\left|\varphi_{k}\left(u_{1}^{n}-u_{2}^{n}\right)\right| d x
\end{gathered}
$$

$$
\begin{aligned}
& \quad \leq \frac{2 l}{k} \int_{\Omega} \Psi_{k}\left(u_{1}^{n}-u_{2}^{n}\right) d x, \\
& -\frac{1}{k} \int_{\Gamma}\left(f\left(u_{1}^{n}\right)-f\left(u_{2}^{n}\right)\right) \varphi_{k}\left(u_{1}^{n}-u_{2}^{n}\right) d S \\
& \quad \leq \frac{l}{k} \int_{\Gamma}\left|u_{1}^{n}-u_{2}^{n}\right|\left|\varphi_{k}\left(u_{1}^{n}-u_{2}^{n}\right)\right| d S \\
& \quad \leq \frac{2 l}{k} \int_{\Gamma} \Psi_{k}\left(u_{1}^{n}-u_{2}^{n}\right) d S,
\end{aligned}
$$

we obtain that

$$
\begin{aligned}
& \frac{1}{k} \frac{d}{d t}\left(\int_{\Omega} \Psi_{k}\left(u_{1}^{n}-u_{2}^{n}\right) d x+\int_{\Gamma} \Psi_{k}\left(u_{1}^{n}-u_{2}^{n}\right) d S\right) \\
& \quad \leq \frac{2 l}{k}\left(\int_{\Omega} \Psi_{k}\left(u_{1}^{n}-u_{2}^{n}\right) d x+\int_{\Gamma} \Psi_{k}\left(u_{1}^{n}-u_{2}^{n}\right) d S\right),
\end{aligned}
$$

which implies that

$$
\begin{aligned}
& \frac{1}{k}\left(\int_{\Omega} \Psi_{k}\left(u_{1}^{n}-u_{2}^{n}\right)(t) d x+\int_{\Gamma} \Psi_{k}\left(u_{1}^{n}-u_{2}^{n}\right)(t) d S\right) \\
& \quad \leq e^{2 l t} \frac{1}{k}\left(\int_{\Omega} \Psi_{k}\left(u_{1,0}^{n}-u_{2,0}^{n}\right) d x\right. \\
& \left.\quad+\int_{\Gamma} \Psi_{k}\left(u_{1,0}^{n}-u_{2,0}^{n}\right) d S\right) .
\end{aligned}
$$

Let $k \longrightarrow 0$. We get

$$
\left\|u_{1}^{n}(t)-u_{2}^{n}(t)\right\|_{L^{1}(\bar{\Omega}, d v)} \leq e^{2 l t}\left\|u_{1,0}^{n}-u_{2,0}^{n}\right\|_{L^{1}(\bar{\Omega}, d v)} .
$$

Therefore, for $n$ large enough we obtain that

$$
\begin{aligned}
& \left\|u_{1,0}^{n}-u_{2,0}^{n}\right\|_{L^{1}(\bar{\Omega}, d v)} \\
& \quad=\left\|u_{1,0}^{n}-u_{1,0}+u_{1,0}-u_{2,0}+u_{2,0}-u_{2,0}^{n}\right\|_{L^{1}(\bar{\Omega}, d v)} \\
& \quad<C\left\|u_{1,0}-u_{2,0}\right\|_{L^{1}(\bar{\Omega}, d v)} .
\end{aligned}
$$

For any $t>0, \varepsilon>0$ and $n$ large enough, we know that there exists $\delta=\varepsilon / C>0$ such that

$$
\left\|u_{1}^{n}(t)-u_{2}^{n}(t)\right\|_{L^{1}(\bar{\Omega}, d v)}<\varepsilon
$$

$$
\text { if }\left\|u_{1,0}-u_{2,0}\right\|_{L^{1}(\bar{\Omega}, d v)}<\delta \text {. }
$$

Since

$$
\begin{aligned}
& \left\|u_{1}(t)-u_{2}(t)\right\|_{L^{1}(\bar{\Omega}, d v)}=\| u_{1}(t)-u_{1}^{n}(t)+u_{1}^{n}(t) \\
& \quad-u_{2}^{n}(t)+u_{2}^{n}(t)-u_{2}(t)\left\|_{L^{1}(\bar{\Omega}, d v)} \leq\right\| u_{1}(t) \\
& \quad-u_{1}^{n}(t)\left\|_{L^{1}(\bar{\Omega}, d v)}+\right\| u_{1}^{n}(t)-u_{2}^{n}(t)\left\|_{L^{1}(\bar{\Omega}, d v)}+\right\| u_{2}^{n}(t) \\
& \quad-u_{2}(t) \|_{L^{1}(\bar{\Omega}, d v)} .
\end{aligned}
$$

In view of (79), for any $\varepsilon>0$ and any fixed $t>0$, we can choose $n$ large enough such that

$$
\left\|u_{i}(t)-u_{i}^{n}(t)\right\|_{L^{1}(\bar{\Omega}, d v)}<\varepsilon / 3, \quad \text { for } i=1,2 .
$$


Therefore, from (87) we deduce that

$$
\begin{aligned}
& \left\|u_{1}(t)-u_{2}(t)\right\|_{L^{1}(\bar{\Omega}, d \nu)}<\varepsilon \\
& \text { as long as }\left\|u_{1,0}-u_{2,0}\right\|_{L^{1}(\bar{\Omega}, d \nu)}<\delta,
\end{aligned}
$$

which means that $S(t)$ is continuous in $L^{1}(\bar{\Omega}, d \nu)$.

Now let us prove that $\{S(t)\}_{t \geq 0}$ has an absorbing set in $L^{1}(\bar{\Omega}, d \nu)$; i.e., for any bounded set $B \subset L^{1}(\bar{\Omega}, d \nu)$, there exist a $T=T(B)$, such that, for all $t \geq T, S(t) B \subset B_{0}\left(\subset L^{1}(\bar{\Omega}, d \nu)\right)$. Repeating the proof of Section 3 Step 1, taking $\varphi_{k}\left(u^{n}\right)$ as a test function in (97), we obtain that

$$
\begin{aligned}
& \frac{d}{d t}\left(\int_{\Omega} \Psi_{k}\left(u^{n}\right)(t) d x+\int_{\Omega} \Psi_{k}\left(u^{n}\right)(t) d S\right) \\
& \quad+\int_{\Omega}\left|\nabla \varphi_{k}\left(u^{n}\right)\right|^{2} d x+\int_{\Gamma}\left|\nabla_{\Gamma} \varphi_{k}\left(u^{n}\right)\right|^{2} d S \\
& \quad+\int_{\Omega} f\left(u^{n}\right) \varphi_{k}\left(u^{n}\right) d x+\int_{\Gamma} f\left(u^{n}\right) \varphi_{k}\left(u^{n}\right) d S \\
& \quad \leq k\|h\|_{L^{1}(\Omega)}+k\|g\|_{L^{1}(\Gamma)} .
\end{aligned}
$$

Similar to the proof of (26), we get

$$
\left\|u^{n}(t)\right\|_{L^{1}(\bar{\Omega}, d v)} \leq \frac{C_{5}}{c_{1}}+e^{-C_{1} t}\left\|u_{0}^{n}\right\|_{L^{1}(\bar{\Omega}, d \nu)}+|\Omega|+|\Gamma|
$$

for any $t>0$.

Passing to the limit, we deduce that

$$
\|u(t)\|_{L^{1}(\bar{\Omega}, d \nu)} \leq \frac{C_{5}}{c_{1}}+e^{-C_{1} t}\left\|u_{0}\right\|_{L^{1}(\bar{\Omega}, d v)}+|\Omega|+|\Gamma|
$$

for any $t>0$.

Thus, the semigroup $\{S(t)\}_{t \geq 0}$ possesses an absorbing set in $L^{1}(\bar{\Omega}, d \nu)$, which can actually be chosen as the ball centered at zero with radius $C_{5} / c_{1}+|\Omega|+|\Gamma|+1$ in $L^{1}(\bar{\Omega}, d \nu)$.

Finally, we prove that $S(t)$ is compact in $L^{1}(\bar{\Omega}, d \nu)$ for any $t>0$; i.e., for any bounded sequence $\left\{u_{0, i}, \gamma\left(u_{0, i}\right)\right\}$, the sequence $\left\{u_{i}(t)\right\}$ has convergent subsequences in $L^{1}(\bar{\Omega}, d \nu)$. Here for every positive integer $i, u_{i}(t)=S(t) u_{0, i}$ denotes the unique entropy solution to the following problem:

$$
\begin{aligned}
\left(u_{i}\right)_{t}-\Delta u_{i}+f\left(u_{i}\right) & =h(x) \quad \text { in } \Omega \times \mathbb{R}^{+}, \\
\left(u_{i}\right)_{t}-\Delta_{\Gamma} u_{i}+\frac{\partial u_{i}}{\partial \nu}+f\left(u_{i}\right) & =g(x) \quad \text { on } \Gamma \times \mathbb{R}^{+} \\
u_{i}(x, 0) & =u_{0, i} \quad \text { in } \Omega .
\end{aligned}
$$

Note, for any $i$, the entropy solution $u_{i}(t)$ of the above problem can be obtained by approximations as in the last section; indeed, consider the following approximate problem for (93):

$$
\begin{aligned}
& \left(u_{i}^{n}\right)_{t}-\Delta u_{i}^{n}+f\left(u_{i}^{n}\right)=h^{n} \quad \text { in } \Omega \times \mathbb{R}^{+}, \\
& \left(u_{i}^{n}\right)_{t}-\Delta_{\Gamma} u_{i}^{n}+\frac{\partial u_{i}^{n}}{\partial \nu}+f\left(u_{i}^{n}\right)=g^{n} \quad \text { on } \Gamma \times \mathbb{R}^{+} \text {, } \\
& u_{i}^{n}(x, 0)=u_{0, i}^{n} \quad \text { in } \Omega,
\end{aligned}
$$

where $h^{n} \in L^{\infty}(\Omega), u_{0, i}^{n} \in L^{\infty}(\Omega), g^{n} \in L^{\infty}(\Gamma)$ converges, respectively, to $h, u_{0, i}, g$ in $L^{1}$-norm, with

$$
\begin{gathered}
\left\|u_{0, i}^{n}\right\|_{L^{1}(\Omega)} \leq\left\|u_{0, i}\right\|_{L^{1}(\Omega)}, \\
\left\|h^{n}\right\|_{L^{1}(\Omega)} \leq\|h\|_{L^{1}(\Omega)}, \\
\left\|g^{n}\right\|_{L^{1}(\Gamma)} \leq\|g\|_{L^{1}(\Gamma)} .
\end{gathered}
$$

Thanks to the analysis in Section 3, we know that $u_{i}^{n}$ satisfies the estimates similar to (26) and (27), i.e.,

$$
\begin{aligned}
& \left\|u_{i}^{n}(t)\right\|_{L^{1}(\bar{\Omega}, d v)} \leq \frac{c_{5}}{c_{1}}+e^{-c_{1} t}\left\|u_{0, i}\right\|_{L^{1}(\bar{\Omega}, d v)}+|\Omega|+|\Gamma| \\
& \text { for any } t>0, \\
& \int_{Q_{T}}\left|\nabla \varphi_{k}\left(u_{i}^{n}\right)\right| d x d t+\int_{\Gamma_{T}}\left|\nabla_{\Gamma} \varphi_{k}\left(u_{i}^{n}\right)\right| d S d t \leq C_{6} k, \\
& \int_{Q_{T}}\left|u_{i}^{n}\right|^{r-1} d x d t+\int_{\Gamma_{T}}\left|u_{i}^{n}\right|^{r-1} d S d t \leq C_{7} .
\end{aligned}
$$

Moreover, up to subsequence $u_{i}^{n}$ converges to the unique entropy solution $u_{i}$ in $C\left(0, T ; L^{1}(\bar{\Omega}, d \nu)\right),\left|u_{i}^{n}\right|^{r-1}$ converges to $\left|u_{i}\right|^{r-1}$ in $L^{1}\left(Q_{T}, d \nu\right)$, and $\nabla \varphi_{k}\left(u_{i}^{n}\right)$ converges to $\nabla \varphi_{k}\left(u_{i}\right)$ in $L^{2}\left(Q_{T}, d \nu\right)$. Passing to the limit in $\mathrm{n}$, we have

$$
\left\|u_{i}(t)\right\|_{L^{1}(\bar{\Omega}, d v)} \leq \frac{C_{5}}{c_{1}}+|\Omega|+|\Gamma|+\left\|u_{0, i}\right\|_{L^{1}(\bar{\Omega}, d v)} e^{-C_{1} t}
$$

for any $t>0$,

and for any fixed $T>0$

$$
\begin{aligned}
& \int_{Q_{T}}\left|\nabla \varphi_{k}\left(u_{i}(\tau)\right)\right| d x d t+\int_{\Gamma_{T}}\left|\nabla_{\Gamma} \varphi_{k}\left(u_{i}(\tau)\right)\right| d S d t \\
& \quad \leq C_{10} k \\
& \int_{Q_{T}}\left|u_{i}(\tau)\right|^{r-1} d x d t+\int_{\Gamma_{T}}\left|u_{i}(\tau)\right|^{r-1} d S d t \leq C_{11} .
\end{aligned}
$$

Here the positive constant $C_{10}, C_{11}$ may depend on $\|h\|_{L^{1}(\Omega)},\|g\|_{L^{1}(\Gamma)},|\Omega|,|\Gamma|, T$ and $L^{1}$-norm of the sequence $\left\{u_{0, i}\right\}$, and they are independent of $i$. Thanks to the estimates (97)-(99), we can obtain that $\left\{\left|\nabla u_{i}\right|^{q_{0}}\right\}$ is bounded in $L^{1}\left(0, T ; L^{1}(\bar{\Omega}, d \nu)\right)$ for any $T>0$, and for any $q_{0} \in \mathbb{R}$ satisfying $1<q_{0}<2(r-1) / r$ in $\bar{\Omega}$, we can deduce that $\left\{u_{i}(t)\right\}$ is bounded in $L^{q_{0}}\left(0, T ; W^{1, q_{0}}(\bar{\Omega}, d \nu)\right)$. Then we deduce from the equation that

$$
\begin{aligned}
& \left\{\left(u_{i}\right)_{t}\right\} \text { is bounded in } L^{q_{0}}\left(0, T ; W^{-1, q_{0}}(\bar{\Omega}, d \nu)\right) \\
& \quad+L^{1}\left(0, T ; L^{1}(\bar{\Omega}, d \nu)\right) .
\end{aligned}
$$

Thanks to the Aubin-Simon type compactness result, as is shown in Lemma 6, there exists a subsequence of $\left\{u_{i}\right\}$, denoted by $\left\{u_{i_{k}}\right\}$ which converges to a function $\tilde{u}$ in $L^{1}\left(0, T ; L^{1}(\bar{\Omega}, d \nu)\right.$, i.e.,

$$
\left\|u_{i_{k}}-\widetilde{u}\right\|_{L^{1}\left(0, T ; L^{1}(\bar{\Omega}, d v)\right)} \longrightarrow 0, \quad \text { as } k \longrightarrow \infty .
$$


Thus, up to a subsequence, $u_{i_{k}}(\tau)$ converges to $\tilde{u}(\tau)$ in $L^{1}(\bar{\Omega}, d \nu)$ for almost all $\tau \in(0, T)$. Especially for any $t>0$, there exist $0<\tau<t$ such that, $u_{i_{k} m}(\tau) \longrightarrow \widetilde{u}$ in $L^{1}(\bar{\Omega}, d \nu)$. Because the map $S(t)$ is continuous map from $L^{1}(\bar{\Omega}, d \nu) \longrightarrow$ $L^{1}(\bar{\Omega}, d \nu)$ we can get

$$
\begin{aligned}
u_{i_{k} m}(t)=S(t-\tau) u_{i_{k} m}(\tau) \longrightarrow S(t-\tau) \tilde{u}(\tau) & \\
& \text { in } L^{1}(\bar{\Omega}, d \nu) .
\end{aligned}
$$

Thus, $S(t)$ is compact in $L^{1}(\bar{\Omega}, d \nu)$. From the standard results on the existence of global attractors [30] we conclude that the semigroup $\{S(t)\}_{t>0}$ possesses a global attractor $\mathscr{A}$ in $L^{1}(\bar{\Omega}, d \nu)$, which is compact, invariant in $L^{1}(\bar{\Omega}, d \nu)$ and attracts every bounded subset of $L^{1}(\bar{\Omega}, d \nu)$ in $L^{1}$-norm.

\section{Data Availability}

No data were used to support this study.

\section{Conflicts of Interest}

The authors declare that they have no conflicts of interest.

\section{Acknowledgments}

This work is supported by NSF of China (11701002) and NSF of Anhui Province (1708085MA02).

\section{References}

[1] M. E. Taylor, Partial differential equations I. Basic theory, vol. 115 of Applied Mathematical Sciences, Springer, New York, NY, USA, 2nd edition, 2011.

[2] J. Escher, "Global existence and nonexistence for semilinear parabolic systems with nonlinear boundary conditions," Mathematische Annalen, vol. 284, no. 2, pp. 285-305, 1989.

[3] A. Miranville and S. Zelik, "Exponential attractors for the Cahn-Hilliard equation with dynamic boundary conditions," Mathematical Methods in the Applied Sciences, vol. 28, no. 6, pp. 709-735, 2005.

[4] G. R. Goldstein, "Derivation and physical interpretation of general boundary conditions," Advances in Differential Equations, vol. 11, no. 4, pp. 457-480, 2006.

[5] A. Miranville, E. Rocca, G. Schimperna, and A. Segatti, "The Penrose-Fife phase-field model with coupled dynamic boundary conditions," Discrete and Continuous Dynamical Systems Series A, vol. 34, no. 10, pp. 4259-4290, 2014.

[6] M. Fila and P. Quittner, "Large time behavior of solutions of a semilinear parabolic equation with a nonlinear dynamical boundary condition," in Topics in nonlinear analysis, vol. 35 of Progress in Nonlinear Differential Equations and Their Applications, pp. 251-272, Birkhäuser, Basel, Switzerland, 1999.

[7] J. M. Arrieta, P. Quittner, and A. b. Rodrguez-Bernal, "Parabolic problems with nonlinear dynamical boundary conditions and singular initial data," Differential and Integral Equations, vol. 14, no. 12, pp. 1487-1510, 2001.

[8] J. L. Vázquez and E. Vitillaro, "Heat equation with dynamical boundary conditions of reactive-diffusive type," Journal of Differential Equations, vol. 250, no. 4, pp. 2143-2161, 2011.
[9] C. G. Gal, "On a class of degenerate parabolic equations with dynamic boundary conditions," Journal of Differential Equations, vol. 253, no. 1, pp. 126-166, 2012.

[10] Z.-H. Fan and C.-K. Zhong, "Attractors for parabolic equations with dynamic boundary conditions," Nonlinear Analysis. Theory, Methods \& Applications, vol. 68, no. 6, pp. 1723-1732, 2008.

[11] C. G. Gal and M. Grasselli, "The non-isothermal Allen-Cahn equation with dynamic boundary conditions," Discrete and Continuous Dynamical Systems - Series A, vol. 22, no. 4, pp. 1009-1040, 2008.

[12] L. Yang and M. Yang, "Long-time behavior of reaction-diffusion equations with dynamical boundary condition," Nonlinear Analysis. Theory, Methods \& Applications, vol. 74, no. 12, pp. 3876-3883, 2011.

[13] C. G. Gal, "The role of surface diffusion in dynamic boundary conditions: Where do we stand?" Milan Journal of Mathematics, vol. 83, no. 2, pp. 237-278, 2015.

[14] C. G. Gal and M. Warma, "Well posedness and the global attractor of some quasi-linear parabolic equations with nonlinear dynamic boundary conditions," Differential and Integral Equations, vol. 23, no. 3-4, pp. 327-358, 2010.

[15] B. You and C. Zhong, "Global attractors for $p$-Laplacian equations with dynamic flux boundary conditions," Advanced Nonlinear Studies, vol. 13, no. 2, pp. 391-410, 2013.

[16] M. a. Anguiano, P. Marín-Rubio, and J. Real, "Pullback attractors for non-autonomous reaction-diffusion equations with dynamical boundary conditions," Journal of Mathematical Analysis and Applications, vol. 383, no. 2, pp. 608-618, 2011.

[17] L. Yang, M. Yang, and P. E. Kloeden, "Pullback attractors for non-autonomous quasi-linear parabolic equations with dynamical boundary conditions," Discrete and Continuous Dynamical Systems - Series B, vol. 17, no. 7, pp. 2635-2651, 2012.

[18] M. a. Anguiano, P. Marín-Rubio, and J. Real, "Regularity results and exponential growth for pullback attractors of a nonautonomous reaction-diffusion model with dynamical boundary conditions," Nonlinear Analysis: Real World Applications, vol. 20, pp. 112-125, 2014

[19] C. Sun and W. Tan, "Non-autonomous reaction-diffusion model with dynamic boundary conditions," Journal of Mathematical Analysis and Applications, vol. 443, no. 2, pp. 1007-1032, 2016.

[20] L. Yang, M. Yang, and J. Wu, "On uniform attractors for nonautonomous $p$-Laplacian equation with a dynamic boundary condition," Topological Methods in Nonlinear Analysis, vol. 42, no. 1, pp. 169-180, 2013.

[21] F. Andreu, N. Igbida, J. M. Mazón, and J. Toledo, "Renormalized solutions for degenerate elliptic-parabolic problems with nonlinear dynamical boundary conditions and $L^{1}$-data," Journal of Differential Equations, vol. 244, no. 11, pp. 2764-2803, 2008.

[22] P. Bénilan, L. Boccardo, T. Gallouët, R. Gariepy, M. Pierre, and J. L. Vázquez, "An $L^{1}$-theory of existence and uniqueness of solutions of nonlinear elliptic equations," Annali della Scuola Normale Superiore di Pisa, vol. 22, no. 2, pp. 241-273, 1995.

[23] A. Prignet, "Existence and uniqueness of 'entropy' solutions of parabolic problems with $L^{1}$ data," Nonlinear Analysis. Theory, Methods \& Applications, vol. 28, no. 12, pp. 1943-1954, 1997.

[24] L. Boccardo and T. Gallouët, "Nonlinear elliptic and parabolic equations involving measure data," Journal of Functional Analysis, vol. 87, no. 1, pp. 149-169, 1989.

[25] X. Chai and W. Niu, "Existence and non-existence results for nonlinear elliptic equations with nonstandard growth," Journal 
of Mathematical Analysis and Applications, vol. 412, no. 2, pp. 1045-1057, 2014.

[26] L. Boccardo and L. Orsina, "Some borderline cases of nonlinear parabolic equations with irregular data," Journal of Evolution Equations (JEE), vol. 16, no. 1, pp. 51-64, 2016.

[27] C. Zhong and W. Niu, "Long-time behavior of solutions to nonlinear reaction diffusion equations involving $L^{1}$ data," Communications in Contemporary Mathematics, vol. 14, no. 1, Article ID 1250007, 19 pages, 2012.

[28] W. Niu and C. Zhong, "Global attractors for the $p$-Laplacian equations with nonregular data," Journal of Mathematical Analysis and Applications, vol. 392, no. 2, pp. 123-135, 2012.

[29] W. Niu, "Global attractors for degenerate semilinear parabolic equations," Nonlinear Analysis. Theory, Methods \& Applications, vol. 77, pp. 158-170, 2013.

[30] J. C. Robinson, Infinite-Dimensional Dynamical Systems: An Introduction to Dissipative parabolic PDEs and the Theory Of Global Attractors, Cambridge Texts in Applied Mathematics, Cambridge University Press, Cambridge, UK, 2001.

[31] J. Simon, "Compact sets in the space $L^{p}(0, T ; B)$," Annali di Matematica Pura ed Applicata, vol. 146, pp. 65-96, 1987.

[32] W. Niu and X. Chai, "Global attractors for nonlinear parabolic equations with nonstandard growth and irregular data," Journal of Mathematical Analysis and Applications, vol. 451, no. 1, pp. 34-63, 2017. 


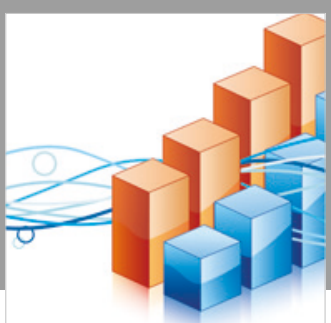

Advances in

Operations Research

\section{-n-m}
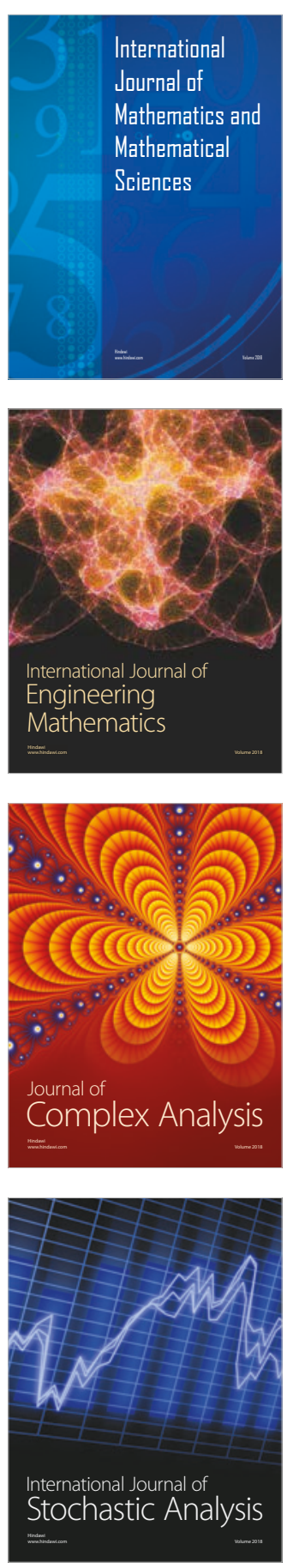
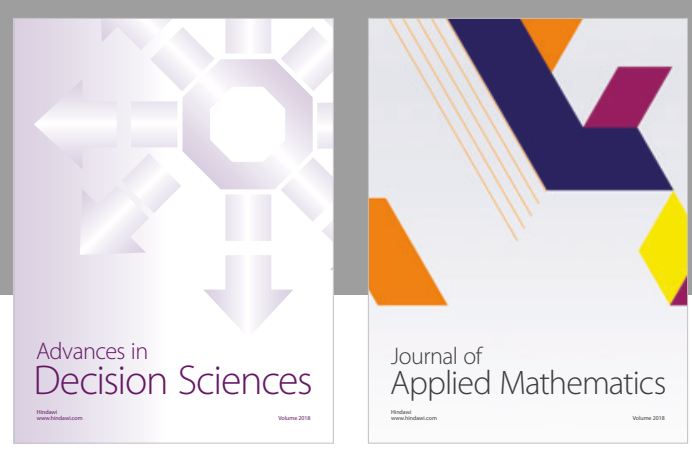

Journal of

Applied Mathematics
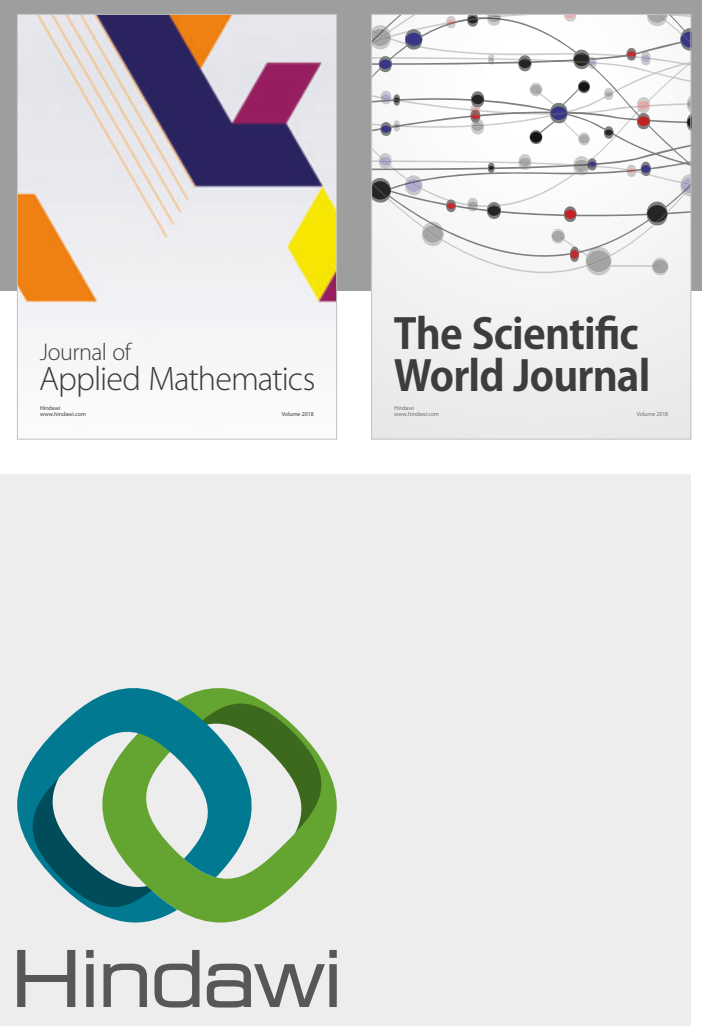

Submit your manuscripts at

www.hindawi.com

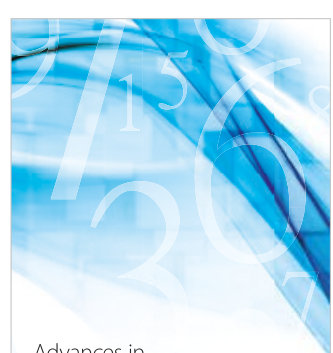

Advances in
Numerical Analysis
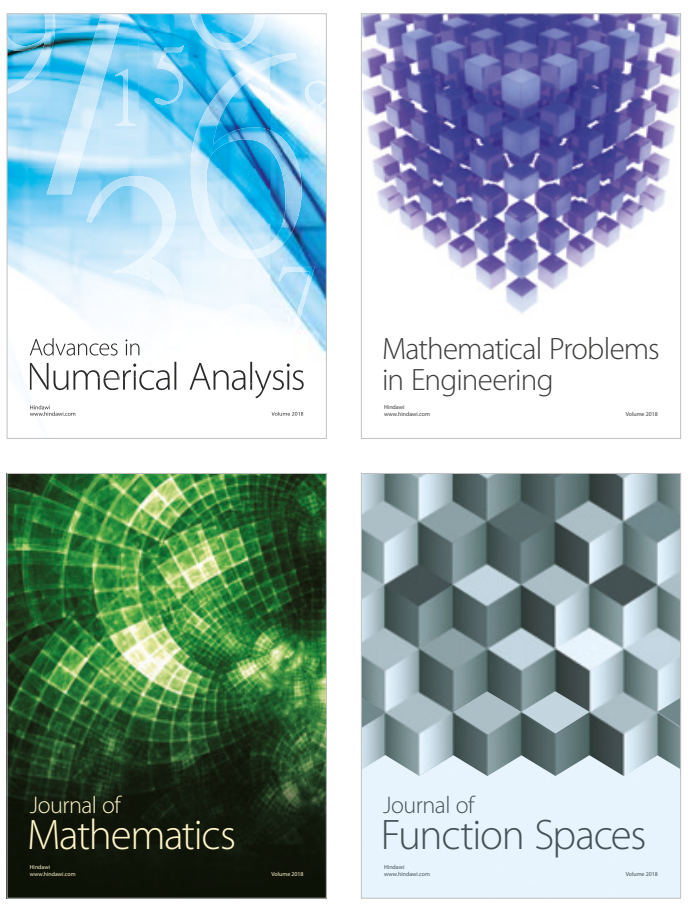

Mathematical Problems in Engineering

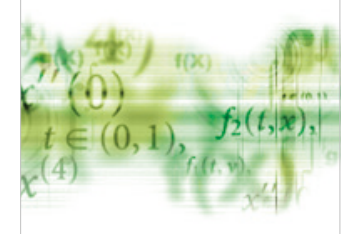

International Journal of

Differential Equations

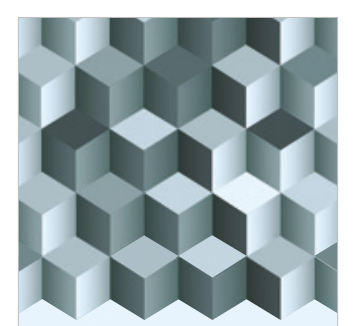

Journal of

Function Spaces

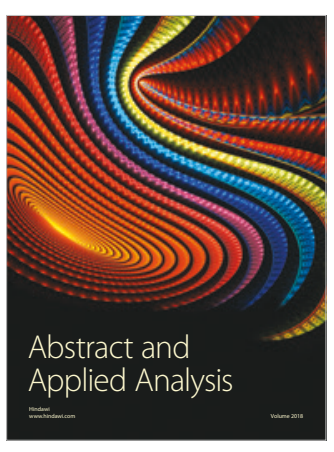

The Scientific

World Journal

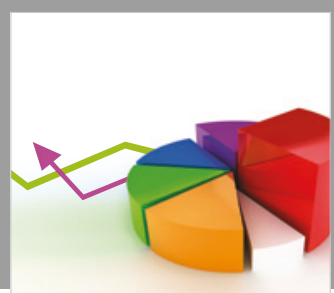

Journal of

Probability and Statistics
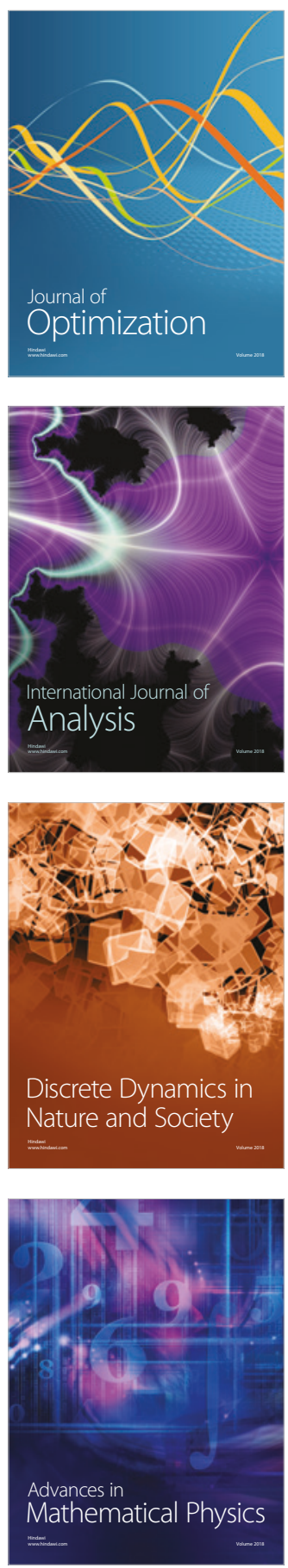